\title{
Evolução de quase-espécies com atribuição não-uniforme de indivíduos
}

\author{
Kádmo de Souza Laxa \\ DISSERTAÇÃO APRESENTADA \\ $\mathrm{AO}$ \\ Instituto DE MATEMÁticA E EstatísticA \\ DA \\ Universidade DE SÃo PAUlo \\ PARA \\ OBTENÇÃO DO TÍTULO \\ $\mathrm{DE}$ \\ Mestre em CiÊnCIAS \\ Programa: Estatística \\ Orientador: Prof. Dr. Luiz Renato Gonçalves Fontes
}

Durante o desenvolvimento deste trabalho o autor recebeu auxílio financeiro do CNPq

São Paulo, Março de 2019 


\section{Evolução de quase-espécies com atribuição não-uniforme de indivíduos}

Esta versão da dissertação contém as correções e alterações sugeridas pela Comissão Julgadora durante a defesa da versão original do trabalho, realizada em 25/02/2019. Uma cópia da versão original está disponível no

Instituto de Matemática e Estatística da Universidade de São Paulo.

Comissão Julgadora:

- Prof. Dr. Luiz Renato Gonçalves Fontes (orientador) - IME-USP

- Prof. Dr. Pablo Martín Rodríguez - ICMC-USP

- Prof. Dr. Alejandro Roldán-Correa - UdeA 


\section{Agradecimentos}

Agradeço primeiramente a Deus, por me proporcionar saúde e força para superar as dificuldades.

A minha família por me incentivar, apoiar, e inspirar. O amor que recebi de cada um foi essencial para seguir na busca pelos meus objetivos.

A todos os professores que me inspiraram e cultivaram em mim o amor pela ciência. Em especial, ao Professor Luiz Renato Gonçalves Fontes, que me auxiliou de forma paciente e se mostrou sempre solícito. 


\section{Resumo}

Laxa, K. S. Evolução de quase-espécies com atribuição não-uniforme de indivíduos. 2019. 29 f. Tese (Mestrado) - Instituto de Matemática e Estatística, Universidade de São Paulo, São Paulo, 2019 .

Recentemente, Ben-Ari e Schinazi (2016) propuseram um modelo estocástico em tempo discreto para a evolução da população de espécies com alta possibilidade de mutação. Neste trabalho, é proposto essencialmente o mesmo modelo, mas em tempo contínuo e com atribuição não-uniforme de indivíduos. Nesse modelo, novas espécies surgem com taxa $r, 0<r<1$, e a cada nova espécie é atribuída, no instante de nascimento, uma aptidão uniforme em $[0,1]$, independente das demais espécies presentes no sistema naquele instante. No instante de surgimento de uma nova espécie, aquela nova espécie passa a contar com 1 indivíduo no sistema. Além disso, com taxa $1-r$ a atribuição de 1 novo indivíduo é feita a uma espécie presente no sistema; cada espécie tem probabilidade proporcional a uma dada função $f$ de sua aptidão de receber o novo indivíduo. Finalmente, com taxa $\mu$, com $\mu<r$, ocorrem eventos de extinção, quando a espécie presente com menor aptidão é removida do sistema (incluindo todos os seus indivíduos). Estudamos o comportamento assintótico da distribuição do tamanho de uma espécie escolhida ao acaso em um tempo grande: determinamos a forma assintótica desta distribuição sob certas condições sobre a função $f$.

Palavras-chave: Modelo de evolução de espécies. Quase-espécies. 


\section{Abstract}

Laxa, K. S. Evolution of quasispecies with non-uniform assignment of individuals. 2019. 29 f. Tese (Mestrado) - Instituto de Matemática e Estatística, Universidade de São Paulo, São Paulo, 2019 .

Recently, Ben-Ari and Schinazi (2016) proposed a discrete time stochastic model for high mutation rate species. Essentially, we propose the same model for the continuous time. In this model, the birth rate of a species is $r, 0<r<1$, and each species receives at birth a uniform fitness in $[0,1]$, independent of the other species of the process at that time. Each new species has 1 individual at the moment of birth. Furthermore, an assignment of a new individual to a species present in the system occurs with rate $1-r$. Each species has a probability of assignment weighted by a given function $f$, that depends on species fitness. Finally, the death rate is $\mu, \mu<r$. When a death event occurs, the species with the lowest fitness of the system is removed with all individuals. We study the asymptotic behavior of the distribution of the size of a species chosen randomly for a big time: we characterize the asymptotic form of this distribution under certain conditions on the function $f$.

Keywords: Evolution of species model. Quasispecies. 


\section{Sumário}

$\begin{array}{ll}\text { Lista de Figuras } & \text { ix }\end{array}$

1 Introdução $\quad \mathbf{1}$

1.1 Construção do modelo BAS . . . . . . . . . . . . . . . . . . . 1

2 Análise do modelo simplificado $\quad 5$

2.1 Descrição do modelo simplificado . . . . . . . . . . . . . . . . . 5

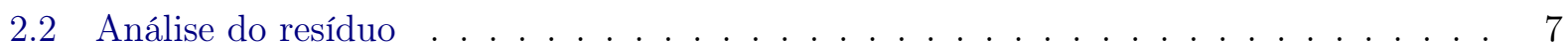

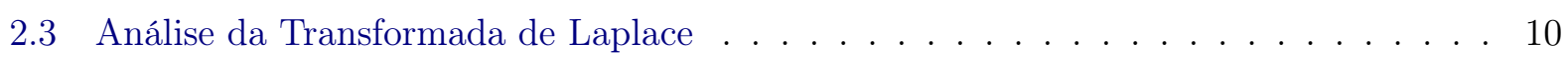

2.3.1 Demonstração do Teorema $2.1 \ldots \ldots \ldots \ldots$. . . . . . . . . . . . 12

3 Análise do modelo BAS $\quad 15$

3.1 Acoplamento para o modelo BAS . . . . . . . . . . . . . . . . 15

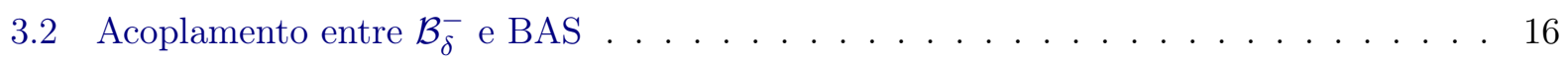

3.2 .1 Construção do conjunto de espécies surgidas . . . . . . . . . . . . . 16

3.2 .2 Construção das extinções de espécies no BAS . . . . . . . . . . . . . . . . . . . . . . . . . . . . . 18

3.2 .3 Atribuição de indivíduos às espécies . . . . . . . . . . . . . . . . . . 18

3.2.4 Sorteio de uma espécie no BAS no tempo t . . . . . . . . . . . . . . . 19

3.3 Acoplamento entre $\mathcal{B}^{+}$e BAS . . . . . . . . . . . . . . . . . . . . . 19

3.3.1 Construção do conjunto das espécies . . . . . . . . . . . . . . . . . . . 19

3.3 .2 Atribuição de indivíduos . . . . . . . . . . . . . . . . . . . 20

3.3 .3 Conclusões . . . . . . . . . . . . . . . . . . . . . . 20

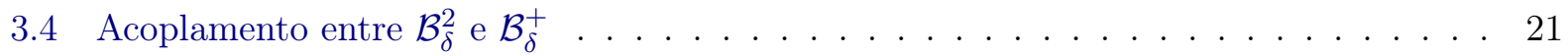

3.5 Demonstrações finais . . . . . . . . . . . . . . . . . . . . . . . . . . . 22

4 Conclusões $\quad 27$

$\begin{array}{ll}\text { Referências Bibliográficas } & 29\end{array}$ 


\section{Lista de Figuras}

2.1 A área azul representa a região em que ocorre o surgimento de espécies e a atribuição de indivíduos no modelo BASS, e o ponto, a espécie adicional. . . . . . . . . . . . 5

3.1 As regiões do plano $[0, t] \times[0,1]$ que são consideradas na construção dos acoplamentos. 16 


\section{Capítulo 1}

\section{Introdução}

\subsection{Construção do modelo BAS}

Uma extensão a tempo contínuo do modelo de Ben Ari \& Schinazi de evolução de quase-espécies apresentado em [BAS16] (Modelo BAS), pode ser descrito informalmente da seguinte forma: o sistema é inicializado com nenhuma espécie; com taxa $r, 0<r<1$, surgem novas espécies no sistema. Cada nova espécie assim surgida conta com um indivíduo no momento do surgimento, e a ela é atribuída, independentemente das demais espécies, uma aptidão, usando dada distribuição contínua, que, sem perda de generalidade, iremos tomar Uniforme em $[0,1]$. Dessa forma, a aptidão caracteriza a espécie, já que espécies diferentes terão aptidões diferentes quase-certamente. Consideramos $\left\{\mathcal{X}_{t}, t \geq 0\right\}$ como sendo o processo de evolução das aptidões das espécies do BAS. Dessa maneira, para $s>0$ fixo, $\mathcal{X}_{s} \subset[0,1]$ é o conjunto das aptidões das espécies presentes no processo no tempo $s$.

Com taxa $1-r$, ocorrem eventos de atribuição de novos indivíduos a espécies presentes no sistema, feita da seguinte forma: em cada evento de atribuição, realizado no tempo $t>0$, quando existem espécies presentes no sistema no tempo $t$ atribui-se um indivíduo a mais a uma espécie presente no sistema escolhida com probabilidade

$$
\frac{f(a)}{F_{t}},
$$

onde $f:(0,1) \rightarrow(0, \infty)$ é dada, $a$ é a aptidão da espécie escolhida e

$$
F_{t}=\sum_{x \in \mathcal{X}_{t}} f(x)
$$

é a soma feita sobre as aptidões de todas as espécies presentes no sistema no momento da atribuição. Cada espécie é contada apenas uma vez na soma, independentemente do número de indivíduos que ela possui no momento da atribuição. Quando não há nenhuma espécie presente no sistema no momento de atribuição de indivíduos o sistema não sofre nenhuma alteração.

Finalmente, há um mecanismo de extinção de espécies, que acontece com taxa $\mu, 0<\mu<r$. A cada momento de extinção, a espécie presente no sistema de menor aptidão é extinta: todos os indivíduos de tal espécie são removidos do sistema.

Denotaremos este modelo por $\operatorname{BAS}([0, \infty),[0,1])$, ou, de maneira mais curta, $\mathcal{B}$. O modelo BAS foi introduzido por Ben-Ari e Schinazi em [BAS16], a tempo discreto e com $f \equiv 1$, com o seguinte resultado: Seja $\eta_{t}$ o número de indivíduos de uma espécie escolhida uniformemente ao acaso entre as espécies presentes no sistema no tempo $t$. Cada espécie é contada apenas uma vez nessa escolha, independentemente do número de indivíduos que ela possui. Definimos

$$
\mu_{c}:=\mu / r \text {. }
$$

Teorema 1.1 (BAS(2016)). Seja $A_{t}$ a aptidão da espécie sorteada uniformemente no tempo $t$ no modelo BAS. Para quase toda realização de variáveis de surgimento, extinção e atribuição, temos 
que quando $t \rightarrow \infty$,

$$
\left(\eta_{t}, A_{t}\right) \rightarrow(G, A)
$$

em distribuição, onde $G \sim$ Geométrica $\left(\frac{\left(1-\mu_{c}\right) r}{(1-r)+\left(1-\mu_{c}\right) r}\right), A \sim$ Uniforme $\left(\mu_{c}, 1\right)$ e G e A são independentes.

No presente trabalho, tomamos $f$ mais geral, e levamos em conta a idade da espécie selecionada no tempo $t$, além de sua aptidão, $A_{t}$. A idade de uma espécie do sistema no tempo $t$ é dada por

$$
I_{t}:=t-S_{t}
$$

onde $S_{t}$ é o tempo de surgimento do primeiro indivíduo da espécie.

Teorema 1.2. Supondo que para algum $\omega \in\left(0, \mu_{c}\right)$

$$
\int_{\mu_{c}-\omega}^{1} f(u) d u<\infty
$$

então

$$
\left(\eta_{t}, \frac{I_{t}}{t}, A_{t}\right) \rightarrow(N, U, V) \text {, em distribuição quando } t \rightarrow \infty
$$

onde $U \sim \operatorname{Uniforme}(0,1), V \sim \operatorname{Uniforme}\left(\mu_{c}, 1\right), N \mid\{U=u, V=v\} \sim \operatorname{Poisson}\left(\frac{(1-r) f(v)}{\int_{\mu_{c}}^{1} f(u) d u} \log \left(\frac{1}{u}\right)\right)$.

Note que não fixamos a realização quase-certa das variáveis de surgimento, extinção e atribuição como no resultado em [BAS16]. Notamos que o resultado apresentado por Ben-Ari e Schinazi pode ser recuperado a partir do nosso resultado ao considerar $f$ constante e integrando na idade, apenas diferenciando na já citada realização quase-certa das variáveis. Para dar uma definição construtiva do modelo BAS, vamos representar o surgimento de novas espécies como um processo pontual de Poisson $\mathcal{N}$ em $[0, \infty) \times[0,1]$ de taxa $r \in(0,1)$ em que cada ponto representa o surgimento de uma nova espécie; a primeira coordenada representando o instante de surgimento, e a segunda coordenada representando a aptidão da espécie surgida.

Os instantes de atribuição de um novo indivíduo à uma espécie são representados por um Processo pontual de Poisson $\mathcal{R}$ de taxa $1-r$ em $[0, \infty)$. Os eventos de extinção de espécies são representados por um Processo pontual de Poisson $\mathcal{M}$ de taxa $\mu \in(0, r)$ em $[0, \infty)$.

É importante notar que se considerarmos apenas a evolução das espécies do modelo BAS, sem considerar a atribuição de indivíduos das mesmas, temos o modelo GMS proposto por Guiol, Machado e Schinazi em [GMS11] e [GMS13]. No modelo GMS, em cada tempo $n \geq 1$ existe o nascimento de uma espécie com probabilidade $p(0<p<1)$, que recebe uma aptidão uniforme em $[0,1]$, e com probabilidade $1-p$ há a morte da espécie presente no sistema com menor aptidão, caso haja alguma. Guiol, Machado e Schinazi mostram que quando a probabilidade de nascimento é maior que a probabilidade de morte, existe um ponto crítico $f_{c}=(1-p) / p$ de modo que o conjunto de espécies com aptidão maior que o ponto crítico convergem para uma distribuição uniforme, enquanto as espécies com aptidão menor que o ponto crítico desaparecem depois de um tempo finito. Esse resultado pode ser observado a partir da convergência de $A_{t}$ para uma Uniforme $\left(\mu_{c}, 1\right)$ no Teorema 1.2 .

Ben-Ari e Schinazi em [BAS16] consideram o número de indivíduos de cada espécie para modelar a evolução de uma população de vírus com alta taxa de mutação. Um vírus como o HIV tem alta taxa de mutação, e populações virais com tais propriedades podem ser interpretadas como um grupo de genótipos relacionados chamados quase-espécies. Dessa maneira, no modelo BAS cada surgimento de uma nova espécie significa que houve uma mutação, ou seja, um novo genoma surge, e por isso ele possui uma aptidão diferente. A atribuição de um indivíduo representa o surgimento um indivíduo sem mutação a partir dos genomas presentes no sistema, caso haja. Nos eventos de extinção há a extinção de um genoma com todos os seus indivíduos. 
A motivação aqui é apresentar uma outra abordagem para a análise do modelo BAS, que permite obter naturalmente as extensões que apresentamos em nosso resultado. Ben-Ari e Schinazi consideram a variável $U_{n}^{k}(f)$ que conta o número de espécies no tempo $n$ com aptidão menor que $f$ e com $k$ filhos. Inicialmente, é feita a análise do limite dessa quantidade para espécies com um filho, e a partir desse resultado, é tomado um argumento indutivo para número de filhos maiores. As convergência são baseadas na Lei dos grandes números e nas estimativas de grandes desvios. Esse argumento não vale para todos os valores de $f$, em um caso mais geral são utilizados argumentos de martingais e filtragem de uma $\sigma$ - álgebra. A abordagem apresentada no decorrer deste trabalho considera uma versão simplificada do modelo BAS, denominada BASS, e a partir da análise do BASS que é feita no Capítulo 2 e de acoplamentos entre o BAS e versões do BASS apresentados no Capítulo 3, conseguimos demonstrar o resultado. 


\section{Capítulo 2}

\section{Análise do modelo simplificado}

\subsection{Descrição do modelo simplificado}

Vamos usar uma versão simplificada do modelo BAS em nossa análise. Dadas duas constantes $b, 0 \leq b<1$, e $c, 0<c \leq 1$, o modelo BAS simplificado (BASS) tem surgimento de espécies e atribuições de indivíduos à uma espécie presente no sistema como no modelo BAS, com as seguintes diferenças:

1. O processo de surgimento de novas espécies é um processo pontual de Poisson em $[b t, t] \times[c, 1]$ de taxa $r$, denotado $\hat{\mathcal{N}}$, adicionado de um ponto aleatório escolhido uniformemente ao acaso em $[b t, t] \times[c, 1]$, chamado de espécie adicional. No tempo bt o modelo não possui nenhuma espécie.

2. O processo de atribuição de novos indivíduos às espécies, denotado $\hat{\mathcal{R}}$, é um processo pontual de Poisson em $[b t, t]$ de taxa $1-r$. A escolha da espécie que recebe um novo indivíduo é feita de acordo com a distribuição (1.1).

Além disso, no modelo BASS não há extinção de espécies.

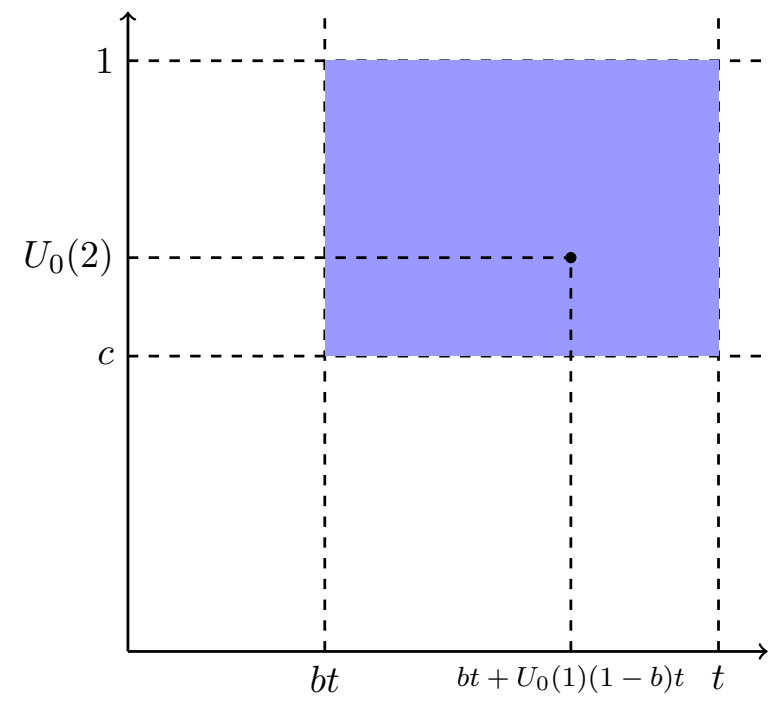

Figura 2.1: A área azul representa a região em que ocorre o surgimento de espécies e a atribuição de indivíduos no modelo BASS, e o ponto, a espécie adicional.

Definimos $\left(\hat{\eta}_{t}, \hat{I}_{t}, \hat{A}_{t}\right)$ como o tamanho, a idade e a aptidão referentes à espécie adicional. Notamos que é possível escrever o instante de surgimento e a aptidão da espécie adicional como

$$
\left(b t+U_{0}(1)(1-b) t, U_{0}(2)\right)
$$


onde $\left(U_{0}(1), U_{0}(2)\right)=U_{0} \sim$ Uniforme $[0,1] \times[c, 1]$ de modo que $U_{0}$ caracteriza a espécie adicional. Vamos enunciar e demonstrar uma versão do Teorema 1.2.

Teorema 2.1. Considerando o modelo BASS e supondo que

$$
\int_{c}^{1} f(u) d u<\infty
$$

então

$$
\left(\hat{\eta}_{t}, \frac{\hat{I}_{t}}{t}, \hat{A}_{t}\right) \rightarrow(\hat{N}, \hat{U}, \hat{V}), \text { em dist. quando } t \rightarrow \infty
$$

onde $\hat{U} \sim \operatorname{Uniforme}(0,1-b), \hat{V} \sim \operatorname{Uniforme}(c, 1) e \hat{N} \mid\{\hat{U}=u, \hat{V}=v\} \sim \operatorname{Poisson}\left(\frac{(1-r) f(v)}{r \int_{c}^{1} f(u) d u} \log \left(\frac{1}{u}\right)\right)$.

Desse modo, considerando $u \in[0,1]$ e $a^{*} \in[c, 1]$ fixos, definimos $\hat{\eta}_{t}\left(u, a^{*}\right)$ como o número de indivíduos da espécie adicional condicionado aos eventos $\left\{\hat{A}_{t}=a^{*}\right\}$ e $\left\{\frac{\hat{I}_{t}}{t}=(1-b)(1-u)\right\}$, ou seja, a espécie adicional surgiu no tempo $b t+(1-b) t u$. Nesse caso,

$$
\hat{\eta}_{t}\left(u, a^{*}\right)=1+\sum_{i=1}^{K} B_{i},
$$

onde $K$ é o número de atribuições no intervalo $[b t+u t(1-b), t]$ e $B_{i}$ a função indicadora de que a i-ésima atribuição tenha sido na espécie adicional; sejam $\left\{T_{i}, i=1, \ldots, K\right\}$ os tempos de atribuição no intervalo $[b t+u t(1-b), t]$. Observamos que

$$
K \sim \operatorname{Poisson}((1-r)(1-u)(1-b) t)
$$

e assim, denotando $\kappa:=(1-r)(1-u)(1-b)$, temos que

$$
\mathbb{E}[K]=\kappa t .
$$

Ainda mais,

$$
T_{i}=b t+u t(1-b)+\sum_{j=1}^{i} R_{j}
$$

onde $R_{j} \sim$ Exponencial $(1-r)$ são independentes e identicamente distribuídas (i.i.d).

Consideramos a sequência de variáveis aleatórias i.i.d. $\left\{A_{1}, A_{2}, \ldots\right\}$, com distribuição Uniforme $(c, 1)$, representando a aptidão das espécies nascidas no intervalo $[b t+u t(1-b), t]$, e assim,

$$
B_{i} \sim \text { Bernoulli }\left(\frac{f\left(a^{*}\right)}{\sum_{j=1}^{\hat{N}_{T_{i}}} f\left(A_{j}\right)+f\left(a^{*}\right)}\right),
$$

onde $\hat{N}_{T_{i}}$ é o número espécies no tempo $T_{i}$, sem contar a espécie adicional, para $i=1, \ldots, K$, ou seja, o número de marcas processo de Poisson $\hat{\mathcal{N}}$ na região $\left[b t, T_{i}\right] \times[c, 1]$. Desse modo, como $\left\{T_{1}, T_{2}, \ldots\right\}$ são independentes de $\hat{\mathcal{N}}$, podemos escrever

$$
\hat{N}_{T_{i}}=\hat{N}_{b t+u t(1-b)}+\sum_{j=1}^{i} L_{j},
$$

onde $L_{i}=\hat{N}_{T_{i}}-\hat{N}_{T_{i-1}} \sim \operatorname{Poisson}\left(\frac{(1-c) r}{1-r}\right)$ i.i.d, com $T_{0}:=b t+u t(1-b)$ e $\hat{N}_{b t+u t(1-b)} \sim$ 
Poisson $(u t(1-c)(1-b) r)$. Desse modo,

$$
\mathbb{E}\left(\hat{N}_{T_{i}}\right)=u t(1-b) \alpha+i\left(\frac{\alpha}{1-r}\right)
$$

em que denotamos $\alpha:=(1-c) r$.

Para analisar $\hat{\eta}_{t}\left(u, a^{*}\right)$ consideramos para $\lambda>0$ a transformada de Laplace de $\hat{\eta}_{t}\left(u, a^{*}\right)$, $\mathbb{E}\left(e^{-\lambda \hat{\eta}_{t}\left(u, a^{*}\right)}\right)$. Para isso, seja $\epsilon>0$ e para $i=1, \ldots, K$ definimos os eventos

$$
\begin{gathered}
D_{i}=\left\{(1-\epsilon) \mathbb{E}\left(\hat{N}_{T_{i}}\right) \leq \hat{N}_{T_{i}} \leq(1+\epsilon) \mathbb{E}\left(\hat{N}_{T_{i}}\right)\right\}, \\
C=\{\mathbb{E}(K)(1-\epsilon) \leq K \leq \mathbb{E}(K)(1+\epsilon)\} \\
E_{i}=\left\{(1-\epsilon) \mathbb{E}\left(f\left(A_{1}\right)\right) \leq \frac{1}{\hat{N}_{T_{i}}} \sum_{j=1}^{\hat{N}_{T_{i}}} f\left(A_{j}\right) \leq(1+\epsilon) \mathbb{E}\left(f\left(A_{1}\right)\right)\right\} .
\end{gathered}
$$

Logo, $\mathbb{E}\left(e^{-\lambda \hat{\eta}_{t}\left(u, a^{*}\right)}\right)$ pode ser decomposta da seguinte maneira:

$$
\begin{aligned}
\mathbb{E}\left(e^{-\lambda \hat{\eta}_{t}\left(u, a^{*}\right)}\right)= & e^{-\lambda} \mathbb{E}\left(e^{-\lambda \sum_{i=1}^{K} B_{i}}\right) \\
= & e^{-\lambda} \mathbb{E}\left(e^{-\lambda \sum_{i=1}^{K} B_{i}} \mid \bigcap_{i=1}^{K} D_{i}, C, \bigcap_{i=1}^{K} E_{i}\right) \mathbb{P}\left(\bigcap_{i=1}^{K} D_{i}, C, \bigcap_{i=1}^{K} E_{i}\right) \\
& +e^{-\lambda} \mathbb{E}\left(e^{-\lambda \sum_{i=1}^{K} B_{i}} \mid \bigcup_{i=1}^{K}\left\{D_{i}^{c} \cup C^{c} \cup E_{i}^{c}\right\}\right) \mathbb{P}\left(\bigcup_{i=1}^{K}\left\{D_{i}^{c} \cup C^{c} \cup E_{i}^{c}\right\}\right) \\
= & e^{-\lambda} \mathbb{E}\left(e^{-\lambda \sum_{i=1}^{K} B_{i}} \mid D, C, E\right) \mathbb{P}(D, C, E)+\varepsilon_{t},
\end{aligned}
$$

em que denotamos

$$
D:=\bigcap_{i=1}^{K} D_{i}, E:=\bigcap_{i=1}^{K} E_{i}
$$

Ainda mais, como $\mathbb{E}\left(e^{-\lambda \sum_{i=1}^{K} B_{i}} \mid \bigcup_{i=1}^{K}\left\{D_{i}^{c} \cup C^{c} \cup E_{i}^{c}\right\}\right) \leq 1$ quando $\lambda>0$, o resíduo $\varepsilon_{t}$ satisfaz

$$
\left|\varepsilon_{t}\right| \leq \mathbb{P}\left(\bigcup_{i=1}^{K}\left\{D_{i}^{c} \cup C^{c} \cup E_{i}^{c}\right\}\right)=\mathbb{P}\left(C^{c}\right)+\mathbb{P}\left(D^{c}, C\right)+\mathbb{P}\left(E^{c}, D, C\right) .
$$

Notamos que

$$
\begin{aligned}
\mathbb{E}\left(\sum_{j=1}^{\hat{N}_{T_{i}}} f\left(A_{j}\right)\right) & =\mathbb{E}\left(\hat{N}_{T_{i}}\right) \mathbb{E}\left(f\left(A_{j}\right)\right)=\left(u t(1-b) \alpha+\frac{i \alpha}{1-r}\right) \int_{c}^{1} \frac{f(x)}{1-c} d x \\
& =\left(u t(1-b)+\frac{i}{1-r}\right) r \int_{c}^{1} f(x) d x
\end{aligned}
$$

Além disso, denotamos

$$
I(c, 1):=r \int_{c}^{1} f(x) d x
$$

\subsection{Análise do resíduo}

Mostraremos a seguir que os termos do resíduo $\varepsilon_{t}$ em (2.10) convergem para 0 quando $t$ diverge. 
Observação 2.2. Pela desigualdade de Chebyshev,

$$
\mathbb{P}\left(C^{c}\right)=\mathbb{P}(|K-\kappa t|>\epsilon \kappa t) \leq \frac{\kappa t}{(\epsilon \kappa t)^{2}} \rightarrow 0,
$$

quando $t \rightarrow \infty$.

Lema 2.1. $\mathbb{P}\left(D^{c}, C\right) \rightarrow 0$, quando $t \rightarrow \infty$.

Demonstração. Pela Lei da probabilidade total, segue que

$$
\begin{aligned}
\mathbb{P}\left(D^{c}, C\right) & \leq \mathbb{P}\left(\bigcup_{i=1}^{K} D_{i}^{c},\{K \leq(1+\epsilon) k t\}\right) \\
& \leq \mathbb{P}\left(\bigcup_{i=1}^{\lfloor k t(1+\epsilon)\rfloor}\left\{\hat{N}_{T_{i}} \geq(1+\epsilon) \mathbb{E}\left(\hat{N}_{T_{i}}\right)\right\}\right)+\mathbb{P}\left(\bigcup_{i=1}^{\lfloor k t(1+\epsilon)\rfloor}\left\{\hat{N}_{T_{i}} \leq(1-\epsilon) \mathbb{E}\left(\hat{N}_{T_{i}}\right)\right\}\right)
\end{aligned}
$$

Para o primeiro elemento da soma de (2.13) temos que

$$
\mathbb{P}\left(\bigcup_{i=1}^{\lfloor k t(1+\epsilon)\rfloor}\left\{\hat{N}_{T_{i}} \geq(1+\epsilon) \mathbb{E}\left(\hat{N}_{T_{i}}\right)\right\}\right) \leq\lfloor k t(1+\epsilon)\rfloor \max _{1 \leq i \leq\lfloor k t(1+\epsilon)\rfloor} \mathbb{P}\left(\left\{\hat{N}_{T_{i}} \geq(1+\epsilon) \mathbb{E}\left(\hat{N}_{T_{i}}\right)\right\}\right) .
$$

Como $\mathbb{E}\left(\hat{N}_{T_{i}}\right)$ é dada por $(2.5)$, temos para $\theta>0$,

$$
\begin{aligned}
\mathbb{P}\left(\hat{N}_{T_{i}} \geq(1+\epsilon) \mathbb{E}\left(\hat{N}_{T_{i}}\right)\right) & =\mathbb{P}\left(\hat{N}_{T_{i}} \geq(1+\epsilon)\left[u t(1-b) \alpha+\frac{i \alpha}{1-r}\right]\right) \\
& =\mathbb{P}\left(e^{\theta \hat{N}_{T_{i}}} \geq e^{\theta(1+\epsilon)\left[u t(1-b) \alpha+\frac{i \alpha}{1-r}\right]}\right) \\
& \leq e^{-\theta(1+\epsilon)\left[u t(1-b) \alpha+\frac{\alpha}{1-r}\right]} \mathbb{E}\left(e^{\theta \hat{N}_{T_{i}}}\right)
\end{aligned}
$$

onde na última passagem é utilizada a desigualdade de Markov. Como $\hat{N}_{T_{i}} \mid T_{i} \sim \operatorname{Poisson}\left(\alpha\left(T_{i}-b t\right)\right)$, temos que sua função geradora existe para todo $\theta \in \mathbb{R}$ e tem a forma

$$
\mathbb{E}\left(e^{\theta \hat{N}_{T_{i}}}\right)=\mathbb{E}\left(e^{\alpha\left(T_{i}-b t\right)\left(e^{\theta}-1\right)}\right) .
$$

Denotando $\gamma:=\left(e^{\theta}-1\right)$, segue que

$$
\begin{aligned}
\mathbb{E}\left(e^{\theta \hat{N}_{T_{i}}}\right) & =\mathbb{E}\left(e^{\gamma \alpha\left(u(1-b) t+\sum_{j=1}^{i} R_{j}\right)}\right)=e^{\gamma \alpha u(1-b) t} \mathbb{E}\left(e^{\left.\gamma \alpha \sum_{j=1}^{i} R_{j}\right)}\right) \\
& =e^{\gamma \alpha u(1-b) t} \mathbb{E}\left(e^{\gamma \alpha R_{1}}\right)^{i}=e^{\gamma \alpha u(1-b) t+i \log \phi_{R}(\gamma \alpha)},
\end{aligned}
$$

onde $\phi_{R}(\gamma \alpha)$ é a função geradora de momentos de $R_{1} \sim \operatorname{Exponencial}(1-r)$ avaliada em $\gamma \alpha$. Assim,

$$
\begin{aligned}
& \mathbb{P}\left(\hat{N}_{T_{i}} \geq(1+\epsilon) \mathbb{E}\left(\hat{N}_{T_{i}}\right)\right) \leq e^{-\theta(1+\epsilon)\left[u t(1-b) \alpha+i\left(\frac{\alpha}{1-r}\right)\right]} e^{\gamma \alpha u(1-b) t+i \log \phi_{R}(\gamma \alpha)} \\
& =\exp (\alpha u t(1-b)[-\theta(1+\epsilon)+\gamma]) \exp \left(i\left[\frac{-(1+\epsilon) \theta \alpha}{1-r}+\frac{\gamma \alpha \log \phi_{R}(\gamma \alpha)}{\gamma \alpha}\right]\right) .
\end{aligned}
$$

Notamos que

$$
\begin{aligned}
& \lim _{x \rightarrow 0} e^{x}-1-(1+\epsilon) x=0 \\
& \left.\frac{d}{d x}\left(e^{x}-1-(1+\epsilon) x\right)\right|_{x=0}=e^{x}-\left.(1+\epsilon)\right|_{x=0}=-\epsilon<0,
\end{aligned}
$$


e assim, para todo $\epsilon>0$, existe $\theta_{1}>0$ tal que $c=e^{\theta_{1}}-1-\theta_{1}(1+\epsilon)<0$. Ainda mais, pelas propriedades da função geradora de momentos, para todo $\epsilon>0$, existe $\alpha_{2}=\left(e^{\theta_{2}}-1\right)>0$ tal que

$$
\frac{\log \phi_{R}\left(\gamma \alpha_{2}\right)}{\gamma \alpha_{2}} \leq \frac{\phi_{R}^{\prime}(0)}{\phi_{R}(0)}(1+\epsilon / 2)=\mathbb{E}\left(R_{1}\right)(1+\epsilon / 2)=\frac{(1+\epsilon / 2)}{1-r}
$$

Desse modo,

$$
i\left[\frac{-(1+\epsilon) \theta \alpha_{2}}{1-r}+\frac{\gamma \alpha_{2} \log \phi_{R}\left(\gamma \alpha_{2}\right)}{\gamma \alpha_{2}}\right] \leq \frac{i \alpha_{2}}{1-r}\left[-\theta_{2}(1+\epsilon)+\gamma(1+\epsilon / 2)\right]
$$

e pelos mesmo argumentos anteriores, temos que

$$
\left.\frac{d}{d x}\left(\left(e^{x}-1\right)(1+\epsilon / 2)-(1+\epsilon) x\right)\right|_{x=0}=-\epsilon / 2<0 .
$$

Assim, para todo $\epsilon>0$, existe $\theta_{2}>0$ tal que

$$
c^{\prime}=i\left[\frac{-(1+\epsilon) \theta_{2} \alpha_{2}}{1-r}+\frac{\gamma \alpha_{2} \log \phi_{R}\left(\gamma \alpha_{2}\right)}{\gamma \alpha_{2}}\right]<0 .
$$

Para qualquer $\theta \leq \min \left\{\theta_{1}, \theta_{2}\right\}$ o limite quando $t \rightarrow \infty$ será

$$
\lim _{t \rightarrow \infty} \mathbb{P}\left(\bigcup_{i=1}^{\lfloor k t(1+\epsilon)\rfloor}\left\{\hat{N}_{T_{i}} \geq(1+\epsilon) \mathbb{E}\left(\hat{N}_{T_{i}}\right)\right\}\right) \leq \lim _{t \rightarrow \infty}\lfloor k t(1+\epsilon)\rfloor e^{t \alpha u(1-\delta) c} e^{c^{\prime}}=0 .
$$

Aplicando os mesmos métodos no segundo elemento da soma de (2.13), temos

$$
\mathbb{P}\left(\bigcup_{i=1}^{\lfloor k t(1+\epsilon)\rfloor}\left\{\hat{N}_{T_{i}} \leq(1-\epsilon) \mathbb{E}\left(\hat{N}_{T_{i}}\right)\right\}\right) \leq\lfloor k t(1+\epsilon)\rfloor \max _{1 \leq i \leq\lfloor k t(1+\epsilon)\rfloor} \mathbb{P}\left(\left\{\hat{N}_{T_{i}} \leq(1-\epsilon) \mathbb{E}\left(\hat{N}_{T_{i}}\right)\right\}\right) .
$$

Tomando $\theta>0$,

$$
\begin{aligned}
\mathbb{P}\left(\hat{N}_{T_{i}} \leq(1-\epsilon) \mathbb{E}\left(\hat{N}_{T_{i}}\right)\right) & =\mathbb{P}\left(\hat{N}_{T_{i}} \leq(1-\epsilon)\left[u t(1-b) \alpha+\frac{i \alpha}{1-r}\right]\right) \\
& =\mathbb{P}\left(e^{\left.-\theta \hat{N}_{T_{i}} \geq e^{-\theta(1-\epsilon)\left[u t(1-b) \alpha+\frac{i \alpha}{1-r}\right]}\right)}\right. \\
& \leq e^{\theta(1-\epsilon)\left[u t(1-b) \alpha+\frac{i \alpha}{1-r}\right]} \mathbb{E}\left(e^{-\theta \hat{N}_{T_{i}}}\right) .
\end{aligned}
$$

Utilizando (2.14) e definindo $\gamma:=\left(1-e^{-\theta}\right)$,

$$
\mathbb{E}\left(e^{-\theta \hat{N}_{T_{i}}}\right)=e^{-\gamma(1-b) u \alpha t+i \log \phi_{R}(-\gamma \alpha)} .
$$

Assim,

$$
\begin{aligned}
& \mathbb{P}\left(\hat{N}_{T_{i}} \leq(1-\epsilon) \mathbb{E}\left(\hat{N}_{T_{i}}\right)\right) \leq e^{\theta(1-\epsilon)\left[u t(1-b) \alpha+\frac{i \alpha}{1-r}\right]} e^{-\gamma(1-b) u \alpha t+i \log \phi_{R}(-\gamma \alpha)} \\
& \leq \exp \left(\alpha u t(1-b)(\theta(1-\epsilon)-\gamma) \exp \left(i \alpha\left(\frac{\theta(1-\epsilon)}{1-r}-\frac{\gamma \log \phi_{R}(-\gamma \alpha)}{-\alpha \gamma}\right)\right)\right.
\end{aligned}
$$


Notamos que

$$
\begin{aligned}
& \lim _{x \rightarrow 0} e^{-x}-1+(1-\epsilon) x=0 \\
& \left.\frac{d}{d x}\left(e^{-x}-1+(1-\epsilon) x\right)\right|_{x=0}=-e^{-x}+\left.(1-\epsilon)\right|_{x=0}=-\epsilon<0,
\end{aligned}
$$

e dessa maneira, analogamente ao caso anterior, para todo $\epsilon>0$, existe $\theta>0, k<0$ e $k^{\prime}<0$ satisfazendo

$$
\lim _{t \rightarrow \infty} \mathbb{P}\left(\bigcup_{i=1}^{\lfloor\kappa t(1+\epsilon)\rfloor}\left\{\hat{N}_{T_{i}} \leq(1-\epsilon) \mathbb{E}\left(\hat{N}_{T_{i}}\right)\right\}\right) \leq \lim _{t \rightarrow \infty}\lfloor\kappa t(1+\epsilon)\rfloor e^{t\left(1-\mu_{c}\right) u k} e^{k^{\prime}}=0 .
$$

Uma vez que mostramos que todos os termos de (2.13) convergem para 0 quando $t$ diverge, concluímos a demonstração.

Lema 2.2. $\mathbb{P}\left(E^{c}, D, C\right) \rightarrow 0$ quando $t \rightarrow \infty$.

Demonstração. Inicialmente, temos que

$$
\begin{aligned}
\mathbb{P}\left(E^{c}, D, C\right) & \leq \mathbb{P}\left(\bigcup_{i=1}^{\lfloor\kappa t(1+\epsilon)\rfloor}\left\{\left|\frac{1}{\hat{N}_{T_{i}}} \sum_{j=1}^{\hat{N}_{T_{i}}} f\left(A_{j}\right)-\mathbb{E}\left(f\left(A_{1}\right)\right)\right|>\epsilon\right\}, \hat{N}_{T_{i}}>(1-\epsilon) \mathbb{E}\left(\hat{N}_{T_{i}}\right)\right) \\
& \leq \mathbb{P}\left(\left\{\left|\frac{1}{n} \sum_{j=1}^{n} f\left(A_{j}\right)-\mathbb{E}\left(f\left(A_{1}\right)\right)\right|>\epsilon\right\}, \text { para algum } n>(1-\epsilon) \mathbb{E}\left(\hat{N}_{b t+u t(1-b)}\right)\right)
\end{aligned}
$$

Assim, tomando o limite quando $t \rightarrow \infty$,

$$
\begin{aligned}
& \mathbb{P}\left(\left\{\left|\frac{1}{n} \sum_{j=1}^{n} f\left(A_{j}\right)-\mathbb{E}\left(f\left(A_{1}\right)\right)\right|>\epsilon\right\}, \text { para algum } n>(1-\epsilon) \mathbb{E}\left(\hat{N}_{b t+u t(1-b)}\right)\right) \rightarrow \\
& \mathbb{P}\left(\bigcap_{n=0}^{\infty}\left\{\left|\frac{1}{n} \sum_{j=1}^{n} f\left(A_{j}\right)-\mathbb{E}\left(f\left(A_{1}\right)\right)\right|>\epsilon\right\}\right) \leq \mathbb{P}\left(\left|\frac{1}{n} \sum_{j=1}^{n} f\left(A_{j}\right)-\mathbb{E}\left(f\left(A_{1}\right)\right)\right| \nrightarrow 0\right)=0,
\end{aligned}
$$

pela Lei dos grandes números, uma vez que assumimos (2.1).

Unindo os Lemas 2.1 e 2.2, concluímos que $\lim _{t \rightarrow \infty} \varepsilon_{t}=0$.

\subsection{Análise da Transformada de Laplace}

Essa seção terá foco na análise do termo

$$
\mathbb{E}\left(e^{-\lambda \sum_{i=1}^{K} B_{i}} \mid D, C, E\right) .
$$

Denotamos

$$
S_{i}=\sum_{j=1}^{\hat{N}_{T_{i}}} f\left(A_{j}\right)
$$


e assim, pela Lei da expectativa total,

$$
\begin{aligned}
& \mathbb{E}\left(e^{-\lambda \sum_{i=1}^{K} B_{i}} \mid D, C, E\right) \\
= & \mathbb{E}\left[\mathbb{E}\left(e^{-\lambda \sum_{i=1}^{K} B_{i}} \mid\left\{\hat{N}_{T_{i}}, i=1, \ldots, K\right\}, K\right) \mid D, C, E\right] \\
= & \mathbb{E}\left[\prod_{i=1}^{K}\left(1-\frac{f\left(a^{*}\right)}{\sum_{j=1}^{\hat{N}_{T_{i}}} f\left(A_{j}\right)+f\left(a^{*}\right)}+\frac{e^{-\lambda} f\left(a^{*}\right)}{\sum_{j=1}^{\hat{N}_{T_{i}}} f\left(A_{j}\right)+f\left(a^{*}\right)}\right) \mid D, C, E\right] \\
= & \mathbb{E}\left[\exp \left(\sum_{i=1}^{K} \log \left(1-\frac{f\left(a^{*}\right)\left(1-e^{-\lambda}\right)}{S_{i}+f\left(a^{*}\right)}\right)\right) \mid D, C, E\right]
\end{aligned}
$$

Notamos agora que, como $\log \left(1-\frac{f\left(a^{*}\right)\left(1-e^{-\lambda}\right)}{S_{i}+f\left(a^{*}\right)}\right)<0$, uma vez que $\lambda>0$, condicionado ao evento $\{D \cap C \cap E\}$, a expressão no interior do sinal da esperança é limitada inferior e superiormente por

$$
\exp \left(\sum_{i=1}^{(1 \mp \epsilon) \kappa} \log \left(1-\frac{f\left(a^{*}\right)\left(1-e^{-\lambda}\right)}{(1 \pm \epsilon) \mathbb{E}\left(\hat{N}_{T_{i}}\right)+f\left(a^{*}\right)}\right)\right)
$$

onde o termo mais acima de "Ғ" ou " \pm " em (2.17) é o termo referente ao limite superior, e o termo mais abaixo é referente ao limite inferior.

Lema 2.3. Para cada $\epsilon>0$ fixo,

$$
\begin{aligned}
\exp \left(-\left(1-e^{-\lambda}\right) \frac{f\left(a^{*}\right)(1-r)}{(1-\epsilon)^{3} I(c, 1)} \log \left(\frac{1+\epsilon(1-u)}{u}\right)\right) & \leq \liminf _{t \rightarrow \infty} \mathbb{E}\left(e^{-\lambda \sum_{i=1}^{K} B_{i}} \mid D, C, E\right) \\
& \leq \limsup _{t \rightarrow \infty} \mathbb{E}\left(e^{-\lambda \sum_{i=1}^{K} B_{i}} \mid D, C, E\right) \\
& \leq \exp \left(-\left(1-e^{-\lambda}\right) \frac{f\left(a^{*}\right)(1-r)}{(1+\epsilon)^{3} I(c, 1)} \log \left(\frac{1-\epsilon(1-u)}{u}\right)\right) .
\end{aligned}
$$

Demonstração. Durante essa demonstração denotaremos $I$ no lugar do termo $I(c, 1)$. Usando o fato de que, para todo $x>0$ suficientemente próximo de zero, vale $-(1-\epsilon) x \leq \log (1-x) \leq-x$, obtemos cotas inferiores e superiores para (2.15) válidas para todo $t$ bastante grande da forma

$$
\exp \left(-\left(1-e^{-\lambda}\right) \sum_{i=1}^{\lfloor\kappa t(1 \mp \epsilon)\rfloor} \frac{f\left(a^{*}\right)}{(1 \pm \epsilon)^{3}\left(u t(1-b)+\frac{i}{1-r}\right) I+(1 \pm \epsilon) f\left(a^{*}\right)}\right)
$$

onde utilizamos (2.5) em (2.21). Ainda mais, a soma em (2.18) tem a forma

$$
\begin{aligned}
& \sum_{i=1}^{\lfloor\kappa t(1 \mp \epsilon)\rfloor} \frac{f\left(a^{*}\right)}{(1 \pm \epsilon)^{3}\left(u t(1-b)+\frac{i}{1-r}\right) I+(1 \pm \epsilon) f\left(a^{*}\right)}=\frac{f\left(a^{*}\right)}{(1 \pm \epsilon)^{3} I} \sum_{i=1}^{\lfloor\kappa t(1 \mp \epsilon)\rfloor} \frac{1}{u t(1-b)+\frac{f\left(a^{*}\right)}{(1 \pm \epsilon)^{2} I}+\frac{i}{1-r}}= \\
& \frac{f\left(a^{*}\right)(1-r)}{(1 \pm \epsilon)^{3} I} \sum_{i=1}^{\lfloor\kappa t(1 \mp \epsilon)\rfloor} \frac{t}{u t(1-b)+\frac{f\left(a^{*}\right)}{(1 \pm \epsilon)^{2} I}+\frac{i}{1-r}} \frac{1}{t(1-r)} \\
& =\frac{f\left(a^{*}\right)(1-r)}{(1 \pm \epsilon)^{3} I} \sum_{i=1}^{\lfloor\kappa t(1 \mp \epsilon)\rfloor} \frac{1}{x_{i}}\left(x_{i+1}-x_{i}\right),
\end{aligned}
$$


onde para $x \in \mathbb{R} \backslash\{0\}$ e $i=0,1, \ldots,\lfloor\kappa t(1+\epsilon)\rfloor$, definimos

$$
x_{i}:=u(1-b)+\frac{f\left(a^{*}\right)}{(1 \pm \epsilon)^{2} I t}+\frac{i}{(1-r) t} .
$$

Assim, como

$$
u(1-b)+\frac{f\left(a^{*}\right)}{(1 \pm \epsilon)^{2} I t}+\frac{\lfloor\kappa t(1 \mp \epsilon)\rfloor}{(1-r) t} \longrightarrow 1-b \mp \epsilon(1-u)(1-b), \text { quando } t \rightarrow \infty,
$$

notamos que (2.19) é uma soma de Riemann da função $1 / x$ no intervalo $[u(1-b), 1-b \mp \epsilon(1-u)(1-b)]$ - vide [Ant99] -, e assim,

$$
\begin{gathered}
\frac{f\left(a^{*}\right)(1-r)}{(1 \pm \epsilon)^{3} I} \sum_{i=1}^{\lfloor\kappa t(1 \mp \epsilon)\rfloor} \frac{1}{x_{i}}\left(x_{i+1}-x_{i}\right) \\
\underset{t \rightarrow \infty}{\longrightarrow} \frac{f\left(a^{*}\right)(1-r)}{(1 \pm \epsilon)^{3} I} \int_{u(1-b)}^{1-b \mp \epsilon(1-u)(1-b)} \frac{1}{x} d x \\
=\frac{f\left(a^{*}\right)(1-r)}{(1 \pm \epsilon)^{3} I} \log \left(\frac{1 \mp \epsilon(1-u)}{u}\right),
\end{gathered}
$$

e assim, aplicando (2.21) em (2.18), terminamos a demonstração.

\subsubsection{Demonstração do Teorema 2.1}

Agora temos as ferramentas necessárias para demonstrar o Teorema 2.1

Demonstração. (Teorema 2.1)

Notamos que

$$
\frac{\hat{I}_{t}}{t}=1-(b+U(1-b))=(1-b)(1-U),
$$

em que $U \sim$ Uniforme $(0,1)$, e assim, para todo $t>0$,

$$
\frac{\hat{I}_{t}}{t} \sim \text { Uniforme }(0,1-b)
$$

Ainda mais, a aptidão é a segunda coordenada de uma Uniforme $[b t, t] \times[c, 1]$, e portanto, $\hat{A}_{t} \sim$ Uniforme $(c, 1)$ para todo $t>0$.

Os Lemas 2.1 e 2.2 mostram que o resíduo converge pra 0 quando $t$ diverge, do que se conclui que

$$
\liminf _{t \rightarrow \infty} \mathbb{E}\left(e^{-\lambda \hat{\eta}_{t}\left(u, a^{*}\right)}\right)=\liminf _{t \rightarrow \infty} e^{-\lambda} \mathbb{E}\left(e^{-\lambda \sum_{i=1}^{K} B_{i}} \mid D, C, E\right),
$$

e o mesmo vale para o limsup. Como o Lema 2.3 vale para qualquer $\epsilon>0$ arbitrário, podemos tomar o limite $\epsilon \rightarrow 0^{+}$, e assim, concluímos que

$$
\lim _{t \rightarrow \infty} \mathbb{E}\left(e^{-\lambda \hat{\eta}_{t}\left(u, a^{*}\right)}\right)=e^{-\lambda} \exp \left(\frac{(1-r)\left(e^{-\lambda}-1\right) f\left(a^{*}\right)}{I(c, 1))} \log \left(\frac{1}{u}\right)\right),
$$

ou seja, $\hat{\eta}_{t}\left(u, a^{*}\right)$ converge em distribuição para uma Poisson $\left(\frac{(1-r) f\left(a^{*}\right)}{I(c, 1)} \log \left(\frac{1}{u}\right)\right)$.

Corolário 2.1. Seja $\hat{A}_{t}$ a aptidão da espécie adicional. Então

$$
\left(\hat{\eta}_{t}, \hat{A}_{t}\right) \rightarrow(\hat{M}, \hat{V}) \text {, em distribuição quando } t \rightarrow \infty,
$$

onde $\hat{V} \sim$ Uniforme $(c, 1)$ e $\hat{M} \mid\{\hat{V}=v\} \sim \operatorname{Geométrica~}\left(\frac{I(c, 1)}{(1-r) f(v)+I(c, 1)}\right)$. 
Demonstração. Notamos que

$$
\lim _{t \rightarrow \infty} \mathbb{E}\left(e^{-\lambda \hat{\eta}_{t}\left(u, a^{*}\right)}\right)=e^{-\lambda} u^{\frac{(1-r)\left(1-e^{-\lambda}\right) f\left(a^{*}\right)}{I(c, 1)}}
$$

e assim,

$$
\begin{aligned}
\lim _{t \rightarrow \infty} \mathbb{E}\left(e^{-\lambda \hat{\eta}_{t}\left(a^{*}\right)}\right) & =\lim _{t \rightarrow \infty} \int_{0}^{1} \mathbb{E}\left(e^{-\lambda \hat{\eta}_{t}\left(u, a^{*}\right)}\right) d u=\int_{0}^{1} \lim _{t \rightarrow \infty} \mathbb{E}\left(e^{-\lambda \hat{\eta}_{t}\left(u, a^{*}\right)}\right) d u \\
& =e^{-\lambda} \int_{0}^{1} u^{\frac{(1-r)\left(1-e^{-\lambda}\right) f\left(a^{*}\right)}{I(c, 1)}} d u=e^{-\lambda} \frac{1}{\frac{(1-r)\left(1-e^{-\lambda}\right)}{I(c, 1)} f\left(a^{*}\right)+1} .
\end{aligned}
$$

Notamos que o limite pode ser trocado com a integral em (2.22) pelo Teorema da convergência dominada, uma vez que $\mathbb{E}\left(e^{-\lambda \hat{\eta}_{t}\left(u, a^{*}\right)}\right)<1$, para qualquer $t>0$.

Denotando $\frac{(1-r) f\left(a^{*}\right)}{I(c, 1)}:=\frac{1-p}{p}$, ou seja,

$$
p:=\frac{I(c, 1)}{(1-r) f\left(a^{*}\right)+I(c, 1)},
$$

temos que

$$
\lim _{t \rightarrow \infty} \mathbb{E}\left(e^{-\lambda \hat{\eta}_{t}\left(a^{*}\right)}\right)=\frac{e^{-\lambda}}{\frac{(1-p)\left(1-e^{-\lambda}\right)}{p}+1}=\frac{p e^{-\lambda}}{1-e^{-\lambda}(1-p)},
$$

e assim, concluímos que $\hat{\eta}_{t}\left(a^{*}\right)$ converge para uma Geométrica $\left(\frac{I(c, 1)}{(1-r) f\left(a^{*}\right)+I(c, 1)}\right)$. 


\section{Capítulo 3}

\section{Análise do modelo BAS}

Para provar o Teorema 1.2, vamos introduzir alguns modelos auxiliares baseados no BASS com os quais estudamos relações de dominação do modelo BAS. A partir destas dominações e dos resultados do Capítulo anterior, o Teorema 1.2 segue rapidamente.

O primeiro modelo, que servirá de "cota inferior" para o BAS será o $B A S S\left([\delta t, t] \times\left[\mu_{c}+\delta, 1\right]\right)$, que denotamos por $\mathcal{B}_{\delta}^{-}$; aqui, $\delta$ é um número arbitrário em $\left(0, \min \left\{1-\mu_{c}, \mu_{c}\right\}\right)$, que será eventualmente levado a 0 . O segundo será o $B A S S\left([0, t] \times\left[\mu_{c}-\delta, 1\right]\right)$, denotado por $\mathcal{B}_{\delta}^{2}$. O terceiro modelo, denotado $\mathcal{B}_{\delta}^{+}$, será $\mathcal{B}_{\delta}^{2}$ unido a um $B A S\left([0, t] \times\left[0, \mu_{c}-\delta\right]\right)$.

\subsection{Acoplamento para o modelo BAS}

Nessa Seção iremos descrever o acoplamento entre o modelo BAS e duas versões do modelo BASS, de modo que exista uma ordem no número de indivíduos no processo com alta probabilidade. Consideramos $\delta>0$ de modo que $\delta<\min \left\{\mu_{c}, 1-\mu_{c}\right\}$. Seja

$$
\mathcal{X}=\left\{\mathcal{X}_{s}, s \geq 0\right\}
$$

o processo de evolução de espécies do modelo BAS. Ou seja, no tempo $s>0, \mathcal{X}_{s}$ é o conjunto das aptidões das espécies presentes no BAS no tempo $s$. Para o modelo $\mathcal{B}_{\delta}^{-}$, definimos analogamente

$$
\mathcal{X}^{-}=\left\{\mathcal{X}_{s}^{-}, s \geq 0\right\}
$$

e para o modelo $\mathcal{B}_{\delta}^{+}$definimos

$$
\mathcal{X}^{+}=\left\{\mathcal{X}_{s}^{+}, s \geq 0\right\}
$$

Os três processos são construídos nos acoplamentos desse capítulo ((3.2), (3.3) e (3.6)). A construção é feita de modo que, com alta probabilidade tenhamos para $s$ grande,

$$
\mathcal{X}_{s}^{-} \subseteq \mathcal{X}_{s} \subseteq \mathcal{X}_{s}^{+}
$$

A construção dos acoplamentos entre os diferentes modelos será feita considerando as seguintes regiões do plano $R:=[0, t] \times[0,1]$ :

$$
\begin{aligned}
& R_{0}:=[0, t] \times\left[0, \mu_{c}-\delta\right] \\
& R_{1}:=[0, \delta t] \times\left[\mu_{c}+\delta, 1\right] \\
& R_{2}:=[\delta t, t] \times\left[\mu_{c}+\delta, 1\right] \\
& R_{3}:=[0, t] \times\left[\mu_{c}-\delta, \mu_{c}+\delta\right] .
\end{aligned}
$$

Essa divisão é feita afim de realizarmos construções distintas nas diferentes partes do plano, de 
maneira conveniente. Ainda mais, definimos duas variáveis aleatórias independentes entre si,

$$
\begin{aligned}
& N_{2} \sim \operatorname{Poisson}\left(r\left|R_{2}\right|\right) ; \\
& N_{13} \sim \operatorname{Poisson}\left(r\left|R_{1} \cup R_{3}\right|\right),
\end{aligned}
$$

onde $\left|R_{2}\right|$ denota a área da região $R_{2}$.

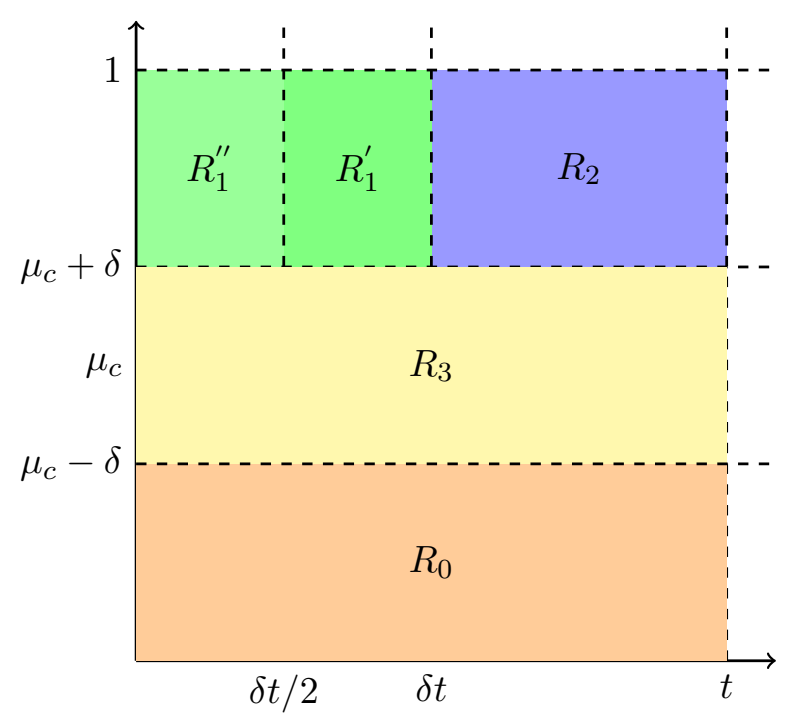

Figura 3.1: As regiões do plano $[0, t] \times[0,1]$ que são consideradas na construção dos acoplamentos.

\subsection{Acoplamento entre $\mathcal{B}_{\delta}^{-}$e BAS}

\subsubsection{Construção do conjunto de espécies surgidas}

Construímos dois processos pontuais de Poisson, um processo pontual de Poisson $\tilde{\mathcal{N}}\left(R_{2}\right)$ em $R_{2}$, e $\tilde{\mathcal{N}}\left(R_{1} \cup R_{3}\right)$ em $R_{1} \cup R_{3}$ descritos da seguinte maneira:

1. Consideramos $U_{0}, U_{1}, U_{2}, \ldots$ uma sequência de variáveis aleatórias i.i.d. e independentes de $N_{2}$ com distribuição Uniforme $\left(R_{2}\right)$. Condicionado ao evento $\left\{N_{2}=n\right\}$ fazemos a seguinte construção:

(a) Se $n=0$, então $\tilde{\mathcal{N}}\left(R_{2}\right)=\emptyset$.

(b) Se $n \geq 1$, então $\tilde{\mathcal{N}}\left(R_{2}\right)=\left\{U_{1}, \ldots, U_{n}\right\}$.

(c) Consideramos um ponto extra $U_{0}$.

2. Consideramos $V_{0}, V_{1}, V_{2}, \ldots$ uma sequência de variáveis aleatórias i.i.d. e independentes de $N_{13}$ com distribuição Uniforme $\left(R_{1} \cup R_{3}\right)$. Condicionado ao evento $\left\{N_{13}=n\right\}$ fazemos a seguinte construção:

(a) Se $n=0$, então $\tilde{\mathcal{N}}\left(R_{1} \cup R_{3}\right)=\emptyset$.

(b) Se $n \geq 1$, então $\tilde{\mathcal{N}}\left(R_{1} \cup R_{3}\right)=\left\{V_{1}, \ldots, V_{n}\right\}$.

(c) Seja

$$
\mathcal{Z} \sim \operatorname{Bernoulli}\left(\frac{\left|R_{2}\right|}{\left|R_{1} \cup R_{2} \cup R_{3}\right|}\right) .
$$

Se $\mathcal{Z}=1, U_{0}$ é a espécie extra. Se $\mathcal{Z}=0$, consideramos $V_{0}$ com sendo a espécie extra. 
Definimos $\tilde{\mathcal{N}}_{123}:=\tilde{\mathcal{N}}\left(R_{2}\right) \cup \tilde{\mathcal{N}}\left(R_{1} \cup R_{3}\right)$. Tais processos de Poisson serão utilizados na construção do conjunto das espécies nascidas do BAS, de $B_{\delta}^{-}$e $B_{\delta}^{+}$.

Seja $\mathcal{N}$ o conjunto dos pares ordenados dos instantes de nascimentos das espécies e suas aptidões no BAS. Definimos $\mathcal{N}^{-}$e $\mathcal{N}^{+}$de maneira análoga para $\mathcal{B}_{\delta}^{-}$e $\mathcal{B}_{\delta}^{+}$. Dividimos a região $R_{1}$ em duas outras regiões,

$$
\begin{aligned}
& R_{1}^{\prime}:=[\delta t / 2, \delta t] \times\left[\mu_{c}+\delta, 1\right], \\
& R_{1}^{\prime \prime}:=[0, \delta t / 2] \times\left[\mu_{c}+\delta, 1\right],
\end{aligned}
$$

e assim, o conjunto de espécies nascidas do $\mathcal{B}^{-}$é dado por

$$
\mathcal{N}^{-}:=\tilde{\mathcal{N}}\left(R_{2}\right),
$$

enquanto o conjunto $\mathcal{N}$ é construído da seguinte maneira:

1. Na região $R_{2}$, a construção é feita da seguinte maneira:

(a) Se $N_{2}=0$, então $\mathcal{N}\left(R_{2}\right)=\emptyset$.

(b) Se $N_{2}=n, n \geq 1$, então consideramos $M \sim \operatorname{Uniforme}(1, \ldots, n)$ e fazemos

$$
\begin{aligned}
& U_{M}^{\prime}=U_{0}, \\
& U_{j}^{\prime}=U_{j}, \text { para } j \neq M .
\end{aligned}
$$

Desse modo, $\mathcal{N}\left(R_{2}\right)=\left\{U_{1}^{\prime}, \ldots, U_{n}^{\prime}\right\}$.

2. Consideramos

$$
N_{1}^{\prime} \sim \operatorname{Poisson}\left(r\left|R_{1}^{\prime}\right|\right)
$$

e também $V_{1}^{\prime}, V_{2}^{\prime}, \ldots$ uma sequência de variáveis aleatórias i.i.d. e independentes de $N_{1}^{\prime}$ com distribuição Uniforme $\left(R_{1}^{\prime}\right)$. O acoplamento na região $R_{1}^{\prime}$ é feita da seguinte maneira:

(a) Se $N_{1}^{\prime}=0$, então $\mathcal{N}\left(R_{1}^{\prime}\right)=\emptyset$.

(b) Se $N_{1}^{\prime}=n, n \geq 1$, então $\mathcal{N}\left(R_{1}^{\prime}\right)=\left\{V_{1}^{\prime \prime}, V_{2}^{\prime}, \ldots, V_{n}^{\prime}\right\}$, onde

$$
V_{1}^{\prime \prime}= \begin{cases}\left(V_{1}^{\prime}(1), U_{0}(2)\right), & \text { se } N_{2}=0, \\ \left(V_{1}^{\prime}(1), U_{M}(2)\right), & \text { caso contrário. }\end{cases}
$$

3. Em $\mathcal{N}\left(R_{0}\right)$, consideramos um processo de Poisson $\tilde{\mathcal{N}}\left(R_{0}\right)$ de taxa $r\left|R_{0}\right|$ independente de $\tilde{\mathcal{N}}$ e em $\mathcal{N}\left(R_{1}^{\prime \prime}\right)$, consideramos um processo de Poisson de taxa $r\left|R_{1}^{\prime \prime}\right|$ independente de $\tilde{\mathcal{N}}$.

Observação 3.1. A construção em $R_{1}^{\prime}$ é feita dessa maneira para que, sempre que $N_{1}^{\prime}>0$, para $s \in[\delta t, t]$, temos que para toda espécie nascida em $B_{\delta}^{-}$até o tempo $s$, existe uma espécie nascida no BAS com a mesma aptidão até o tempo $s$. Essa propriedade será muito importante na construção da atribuição de espécies de maneira acoplada e do sorteio de uma espécie no BAS.

\subsubsection{Construção das extinções de espécies no BAS}

Agora, com o conjunto de especies nascidas no BAS, seja $\mathcal{M}$ um processo pontual de Poisson em $[0, t]$ de taxa $1-r$, em que suas marcas representam os tempos das extinções de espécies no BAS. Sejam $m_{1}, \ldots, m_{n}$ os tempos de extinção em $[0, t]$. Para $0 \leq s_{1} \leq s_{2} \leq t$, definimos

$$
\mathcal{N}_{s_{1}, s_{2}}:=\mathcal{N} \cap\left(\left[s_{1}, s_{2}\right] \times[0,1]\right),
$$


o conjunto de espécies nascidas entre os tempos $s_{1}$ e $s_{2}$. Definimos ainda mais

$$
\mathcal{N}_{s_{1}, s_{2}}^{1}
$$

como o conjunto das aptidões das espécies de $\mathcal{N}_{s_{1}, s_{2}}$. Dessa maneira, definimos

$$
\mathcal{X}_{s}= \begin{cases}\mathcal{N}_{0, s}^{1}, & \text { se } s<m_{1}, \\ \left(\mathcal{X}_{m_{i}^{-}} \backslash \min \left\{\mathcal{X}_{m_{i}^{-}}\right\}\right) \cup \mathcal{N}_{m_{i}, s}^{1}, & \text { se } m_{i} \leq s<m_{i+1} \text { para } i=1, \ldots, n .\end{cases}
$$

onde consideramos $m_{n+1}:=t$. Como no modelo $B A S S$ não há mortes de espécies, definimos

$$
\mathcal{X}_{s}^{-}
$$

como o conjunto da aptidão das espécies de $\mathcal{N}^{-} \cap([0, s] \times[0,1])$. Agora que temos o conjuntos das espécies do BAS e de $\mathcal{B}_{\delta}^{-}$construídos, definimos

$$
\begin{aligned}
& N_{s}:=\left|\mathcal{X}_{s}\right|, \\
& N_{s}^{-}:=\left|\mathcal{X}_{s}^{-}\right|,
\end{aligned}
$$

como sendo o número de espécies vivas no tempo $s$ de cada processo. Definimos adicionalmente para $0 \leq a<b \leq 1$,

$$
\begin{aligned}
& \mathcal{X}_{s}(a, b):=\mathcal{X}_{s} \cap[a, b], \\
& \mathcal{X}_{s}^{-}(a, b):=\mathcal{X}_{s}^{-} \cap[a, b],
\end{aligned}
$$

como sendo o conjunto de aptidões das espécies vivas no tempo $s$ com aptidão no intervalo $[a, b]$, e também

$$
\begin{aligned}
& N_{s}(a, b):=\left|\mathcal{X}_{s}(a, b)\right|, \\
& N_{s}^{-}(a, b):=\left|\mathcal{X}_{s}^{-}(a, b)\right| .
\end{aligned}
$$

\subsubsection{Atribuição de indivíduos às espécies}

Desse modo, com o conjunto de espécies do BAS e de $\mathcal{B}^{-}$, vamos realizar a atribuição de indivíduos às espécies dos modelos. A escolha das espécies é feita de maneira aleatória de acordo com a distribuição (1.1). Para isso, recorremos à definição (1.2) e definimos adicionalmente para $s>0$,

$$
F_{s}^{-}:=\sum_{x \in \mathcal{X}_{t}^{-}} f(x)
$$

e para $0 \leq a<b \leq 1$,

$$
F_{s}(a, b):=\sum_{x \in \mathcal{X}_{s}(a, b)} f(x)
$$

$F_{s}^{-}(a, b)$ é definido de modo análogo a $(3.4)$ para $\mathcal{X}_{s}^{-}(a, b)$.

Para isso, consideramos o evento

$$
G=\left\{N_{s}\left(0, \mu_{c}+\delta\right) \geq 1, \forall s \geq \delta t / 2\right\} .
$$

Seja $\mathcal{R}$ um processo de Poisson de taxa $1-r$ em $[0, t]$ em que suas marcas representam os tempos de atribuição de indivíduos.

1. Quando ocorre um evento de $\mathcal{R}$ no tempo $s \leq t$, existe o evento de atribuição de um indivíduo 
a uma espécie presente no tempo $s$.

(a) Se $s<\delta t$ e $N_{s}>0$, uma espécie de $\mathcal{X}_{s}$ é escolhida aleatoriamente de acordo com a distribuição (1.1) e ela passa a ter mais um indivíduo a partir do tempo $s$. Sempre que $N_{s}=0$, nenhuma mudança ocorre em $\mathcal{X}$, e o mesmo ocorre para $\mathcal{X}^{-}$quando $N_{s}^{-}=0$.

(b) Se $s \geq \delta t, N_{s}>0$ e $N_{s}^{-}>0$ uma espécie de $\mathcal{X}_{s}$ e uma espécie de $\mathcal{X}_{s}^{-}$são escolhidas de maneira acoplada. Em $G \cap\left\{N_{1}^{\prime}>0\right\}$ procedemos da seguinte maneira:

i. Com probabilidade $F_{s}^{-} / F_{s}$ é selecionada uma espécie em $\mathcal{X}_{s}^{-}$de acordo com a distribuição (1.1) e seu tamanho é acrescido em uma unidade nos processos $\mathcal{X}$ e $\mathcal{X}^{-}$.

ii. Com probabilidade $\left(F_{s}-F_{s}^{-}\right) / F_{s}$, é selecionada uma espécie em $\mathcal{X}_{s} \backslash \mathcal{X}_{s}^{-}$e seu tamanho é acrescido em uma unidade em $\mathcal{X}$ e $\mathcal{X}^{+}$. Simultaneamente, é escolhida de maneira independente uma espécie de $\mathcal{X}^{-}$e seu tamanho é acrescido em uma unidade.

(c) $\operatorname{Em}\left(G \cap\left\{N_{1}^{\prime}>0\right\}\right)^{c}$, selecionamos de maneira independente uma espécie em $\mathcal{X}_{s}$ e uma espécie em $\mathcal{X}_{s}^{-}$e cada espécie selecionada recebe um novo indivíduo.

\subsubsection{Sorteio de uma espécie no BAS no tempo $\mathrm{t}$}

Para sorteio de uma espécie no BAS consideramos a variável aleatória $Y$ que supõe $N_{t} \geq 1$, dada por

$$
Y \sim \operatorname{Bernoulli}\left(N_{t}^{-} / N_{t}\right)
$$

1. Se $N_{t}=0$, não existe espécie sorteada.

2. Em $G$, consideramos dois casos.

(a) Se $Y=1$, a espécie sorteada é $U_{0}$.

(b) Se $Y=0$, a espécie sorteada é $U_{L}^{*}$, onde $\left\{U_{1}^{*}, \ldots, U_{N_{t}^{*}}^{*}\right\}$ é uma enumeração de $\mathcal{X}_{t} \backslash \mathcal{X}_{t}^{-}$, $N_{t}^{*}:=\left|\mathcal{X}_{t} \backslash \mathcal{X}_{t}^{-}\right|$e $L \sim \operatorname{Uniforme}\left(1, \ldots, N_{t}^{*}\right)$.

3. Em $G^{c}$, a espécie sorteada é $U_{Q}^{\prime \prime \prime}$, onde $\left\{U_{1}^{\prime \prime \prime}, \ldots, U_{N_{t}}^{\prime \prime \prime}\right\}$ é uma enumeração de $N_{t}$ e $Q \sim$ Uniforme $\left(1, \ldots, N_{t}\right)$.

Caso haja uma espécie sorteada, definimos $\left(\eta_{t}, I_{t}, A_{t}\right)$ como o tamanho, a idade e a aptidão referentes à espécie sorteada. Definimos $\left(\eta_{t}^{-}, A_{t}^{-}, I_{t}^{-}\right)$de maneira análoga para a espécie adicional de $\left\{\mathcal{X}_{t}^{-}, t \geq 0\right\}$.

Observação 3.2. Em $G \cap\{Y=1\}$ temos que a espécie sorteada é a espécie adicional de $\mathcal{B}_{\delta}^{-}$, ou seja,

$$
\left(I_{t}, A_{t}\right)=\left(I_{t}^{-}, A_{t}^{-}\right) \text {. }
$$

\subsection{Acoplamento entre $\mathcal{B}^{+}$e BAS}

\subsubsection{Construção do conjunto das espécies}

Um acoplamento entre as espécies surgidas do modelo BAS e $\mathcal{B}^{+}$é feito da seguinte maneira:

1. Consideramos

$$
\mathcal{N}^{+}\left(R_{1} \cup R_{2} \cup R_{3}\right):=\tilde{\mathcal{N}}_{123},
$$

de modo que a construção na região $R_{2}$ é feita como no acoplamento anterior e $\mathcal{N}\left(R_{1}^{\prime}\right)=\tilde{\mathcal{N}}\left(R_{1}^{\prime}\right)$.

2. Em $\mathcal{N}\left(R_{0}\right)$, consideramos o mesmo processo de Poisson $\tilde{\mathcal{N}}\left(R_{0}\right)$ de taxa $r\left|R_{0}\right|$ utilizado na construção do BAS, de modo que

$$
\mathcal{N}^{+}\left(R_{0}\right)=\tilde{\mathcal{N}}\left(R_{0}\right)
$$


O processo de extinção de espécies no $B_{\delta}^{+}$é acoplado ao processo de extinção de espécies do BAS de modo que todas as espécies extintas no BAS com aptidão menor que $\mu_{c}-\delta$ também sejam extintas no $B_{\delta}^{+}$. Dessa maneira, definimos

$$
\mathcal{X}_{s}^{+}:=\mathcal{X}_{s}^{+}\left(0, \mu_{c}-\delta\right) \cup \mathcal{X}_{s}^{+}\left(\mu_{c}-\delta, 1\right)
$$

onde

$$
\begin{aligned}
& \mathcal{X}_{s}^{+}\left(0, \mu_{c}-\delta\right):=\mathcal{X}_{s}\left(0, \mu_{c}-\delta\right), \\
& \mathcal{X}_{s}^{+}\left(\mu_{c}-\delta, 1\right):=\mathcal{N}_{0, s}^{+, 1}\left(\mu_{c}-\delta, 1\right),
\end{aligned}
$$

onde $\mathcal{N}_{0, s}^{+, 1}$ é definido analogamente a (3.1), como o conjunto da aptidão das espécies de $\mathcal{N}^{+}$nascidas até o tempo $s$ e com aptidões entre $\mu_{c}-\delta$ e 1. Definimos de maneira similar às definições anteriores,

$$
\begin{aligned}
& N_{s}^{+}:=\left|\mathcal{X}_{s}^{+}\right|, \\
& \mathcal{X}_{s}^{+}(a, b):=\mathcal{X}_{s}^{+} \cap[a, b], \\
& N_{s}^{+}(a, b):=\left|\mathcal{X}_{s}^{+}(a, b) \cap[a, b]\right|, \\
& F_{s}^{+}:=\sum_{x \in \mathcal{X}_{s}^{+}} f(x), \\
& F_{s}^{+}(a, b):=\sum_{x \in \mathcal{X}_{s}^{+}(a, b)} f(x) .
\end{aligned}
$$

\subsubsection{Atribuição de indivíduos}

A atribuição de indivíduos é feita de maneira bem simples no acoplamento entre $\mathcal{B}^{+}$e o BAS.

1. Quando ocorre um evento de $\mathcal{R}$ no tempo $s \leq t$, existe o evento de atribuição de um indivíduo à uma espécie já existente. Sempre que $N_{s}=0$, nenhuma mudança ocorre em $\mathcal{X}$, e o mesmo ocorre para $\mathcal{X}^{+}$quando $N_{s}^{+}=0$.

(a) Com probabilidade $F_{s} / F_{s}^{+}$uma espécie em $\mathcal{X}_{s}$ é escolhida aleatoriamente de acordo com a distribuição (1.1) e ela passa a ter mais um indivíduo a partir do tempo $s$ em $\mathcal{X}$ e $\mathcal{X}^{+}$.

(b) Com probabilidade $\left(F_{s}^{+}-F_{s}\right) / F_{s}^{+}$uma espécie em $\mathcal{X}_{s}^{+} \backslash \mathcal{X}_{s}$ é escolhida aleatoriamente de acordo com a distribuição (1.1) e ela passa a ter mais um indivíduo a partir do tempo $s$ em $\mathcal{X}^{+}$. Simultaneamente, é escolhida independentemente uma espécie em $\mathcal{X}$ que passa a ter mais um indivíduo.

\subsubsection{Conclusões}

Considerando a escolha da espécie realizada no acoplamento entre o BAS e $\mathcal{B}_{\delta}^{+}$igual à escolha realizada no acoplamento entre o $\mathrm{BAS}$ e $\mathcal{B}^{-}$, definimos $\left(\eta_{t}^{+}, I_{t}^{+}, A_{t}^{+}\right)$como o tamanho, a idade e a aptidão referentes à espécie adicional de $\mathcal{B}_{\delta}^{+}$. Assim, podemos chegar às seguintes conclusões:

1. Em $G$, temos que nenhuma espécie que surge em $R_{2}$ será extinta, e assim, como $U_{0}=U_{M}^{\prime}$ onde $M \sim$ Uniforme $\left\{1, \ldots, N_{2}\right\}$ e $N_{2}$ é o número de nascimentos do BAS em $R_{2}$, concluímos que o sorteio é uniforme em $N_{t}$ mesmo sem o condicionamento em $Y$.

2. Em $G \cap\{Y=1\}$, a espécie sorteada é $U_{0}$. Ainda mais,

$$
\mathbb{P}(A \cap\{Y=0\}) \leq \mathbb{P}(A) \mathbb{E}\left(\frac{N_{t}\left(0, \mu_{c}-\delta\right)+N_{13}}{N_{13}+N_{2}+N_{t}\left(0, \mu_{c}-\delta\right)}\right) .
$$

Notamos que $N_{t}\left(0, \mu_{c}-\delta\right)$ é um processo de nascimento e morte com taxa de nascimento $r(\mu / r-\delta)$ e taxa de morte $\mu$. Como a taxa de nascimento de $N_{t}\left(0, \mu_{c}-\delta\right)$ é menor que a taxa 
de morte, sabemos que $N_{t}\left(0, \mu_{c}-\delta\right)$ é um processo recorrente, e assim, como veremos com mais detalhes na demonstração do Lema $3.1, N_{t}\left(0, \mu_{c}-\delta\right)$ é dominada estocasticamente por uma variável aleatória finita quase certamente, dada por (3.15), e assim,

$$
\mathbb{P}\left(N_{t}\left(0, \mu_{c}-\delta\right)<\infty\right)=1 .
$$

Ainda mais, pela Lei dos Grandes Números do processo pontual de Poisson,

$$
\frac{N_{13}}{N_{13}+N_{2}} \rightarrow \frac{\left|R_{1}\right|+\left|R_{3}\right|}{\left|R_{1}\right|+\left|R_{2}\right|+\left|R_{3}\right|} \text {, quase certamente quando } t \rightarrow \infty \text {. }
$$

Desse modo, sabemos que existe uma constante $d>0$ fixa, de modo que para todo $\delta>0$ suficientemente pequeno, temos

$$
\frac{\left|R_{1}\right|+\left|R_{3}\right|}{\left|R_{1}\right|+\left|R_{2}\right|+\left|R_{3}\right|}<\delta d
$$

e assim, usando o Teorema da Convergência Dominada concluímos que

$$
\lim _{\delta \rightarrow 0^{+}} \lim _{t \rightarrow \infty} \mathbb{P}\left((G \cap\{Y=1\})^{c}\right)=0 .
$$

3. Notamos que em $G \cap\left\{N_{1}^{\prime} \geq 1\right\}$, para $\delta t \leq s \leq t$, definindo os conjuntos

$$
\begin{aligned}
& L_{1}:=\tilde{\mathcal{N}}\left(R_{2}\right) \cap([\delta t, s] \times[0,1]) \cup\left\{U_{0}\right\}, \\
& L_{2}:=\tilde{\mathcal{N}}_{123} \cup \tilde{\mathcal{N}}\left(R_{0}\right) \cap([0, s] \times[0,1]) \cup\left\{U_{0}\right\},
\end{aligned}
$$

segue que

$$
\mathcal{X}_{s}^{-}=\left\{a: \exists r>0,(r, a) \in L_{1}\right\} \subseteq \mathcal{X}_{s} \subseteq\left\{a: \exists r>0,(r, a) \in L_{2}\right\}=\mathcal{X}_{s}^{+} .
$$

4. Em $G \cap\left\{N_{1}^{\prime} \geq 1\right\} \cap\{Y=1\}$, no acoplamento entre $B^{-}$e o BAS,

$$
\eta_{t}^{-} \geq \eta_{t}
$$

e no acoplamento entre $B^{+}$e o BAS,

$$
\eta_{t} \geq \eta_{t}^{+}
$$

\subsection{Acoplamento entre $\mathcal{B}_{\delta}^{2}$ e $\mathcal{B}_{\delta}^{+}$}

Para a demonstração do Lema 3.1, vamos escrever um acoplamento entre $\mathcal{B}_{\delta}^{2}$ e $\mathcal{B}_{\delta}^{+}$, que é feito de forma simples. A construção de $\mathcal{B}_{\delta}^{+}$é feita como acima. Sendo $\mathcal{N}^{2}$ o conjunto de espécies nascidas em $\mathcal{B}_{\delta}^{2}$, definimos

$$
\mathcal{N}^{2}:=\mathcal{N}^{+} \cap\left([0, t] \times\left[\mu_{c}-\delta, 1\right]\right),
$$

ou seja, as espécies surgidas em $\mathcal{B}_{\delta}^{2}$ são as mesmas espécies surgidas em $\mathcal{B}_{\delta}^{+}$que possuem aptidão maior que $\mu_{c}-\delta . \operatorname{Em~} \mathcal{B}_{\delta}^{2}$ não há extinção de espécies, e assim, sendo

$$
\mathcal{X}^{2}:=\left\{\mathcal{X}_{s}^{2}, s>0\right\}
$$

o processo de evolução das aptidões das espécies de $\mathcal{B}_{\delta}^{+}$, definimos

$$
\mathcal{X}_{s}^{2}:=\mathcal{X}_{s}^{+}\left(\mu_{c}-\delta, 1\right)
$$


Ainda mais, definindo

$$
F_{s}^{2}:=F_{s}^{+}\left(\mu_{c}-\delta, 1\right)
$$

a atribuição de indivíduos é feita da seguinte maneira.

1. Quando ocorre um evento de $\mathcal{R}$ no tempo $s \leq t$, existe o evento de atribuição de um indivíduo a uma espécie já existente. Sempre que $N_{s}^{2}=0$, nenhuma mudança ocorre em $\mathcal{X}^{2}$, e o mesmo ocorre para $\mathcal{X}^{+}$quando $N_{s}^{+}=0$.

(a) Com probabilidade $F_{s}^{2} / F_{s}^{+}$uma espécie em $\mathcal{X}_{s}^{2}$ é escolhida aleatoriamente de acordo com a distribuição (1.1) e ela passa a ter mais um indivíduo a partir do tempo $s$ em $\mathcal{X}^{2}$ e $\mathcal{X}^{+}$.

(b) Com probabilidade $\left(F_{s}^{+}-F_{s}^{2}\right) / F_{s}^{+}$uma espécie em $\mathcal{X}_{s}^{+} \backslash \mathcal{X}_{s}^{2}$ é escolhida aleatoriamente de acordo com a distribuição (1.1) e ela passa a ter mais um indivíduo a partir do tempo $s$ em $\mathcal{X}^{+}$. Simultaneamente, é escolhida independentemente uma espécie em $\mathcal{X}^{2}$ que passa a ter mais um indivíduo.

Definimos $\left(\eta_{t}^{2}, I_{t}^{2}, A_{t}^{2}\right)$ como o tamanho, a idade e a aptidão referentes à espécie adicional de $\mathcal{B}_{\delta}^{2}$.

\subsection{Demonstrações finais}

Lema 3.1. Supondo (1.5),

$$
\left(\eta_{t}^{+}, \frac{I_{t}^{+}}{t}, A_{t}^{+}\right) \rightarrow\left(N^{+}, U^{+}, V^{+}\right) \text {, em distribuição quando } t \rightarrow \infty
$$

para $\delta \in\left[0, \min \left\{\mu_{c}, 1-\mu_{c}\right\}\right)$, onde $U^{+} \sim \operatorname{Uniforme}(0,1), V^{+} \sim \operatorname{Uniforme}\left(\mu_{c}-\delta, 1\right) e$ $N^{+} \mid\left\{U^{+}=u, V^{+}=v\right\} \sim$ Poisson $\left(\frac{(1-r) f(v)}{\int_{\mu_{c}-\delta}^{1} f(u) d u} \log (u)\right)$.

Demonstração. Notamos que no acoplamento apresentado entre $\mathcal{B}_{\delta}^{2}$ e $\mathcal{B}_{\delta}^{+}$na Seção 3.4 , temos que a espécie adicional dos dois processos é igual, ou seja,

$$
\left(I_{t}^{2}, A_{t}^{2}\right)=\left(I_{t}^{+}, A_{t}^{+}\right) .
$$

Desse modo, notamos que $N_{t}^{+}\left(0, \mu_{c}-\delta\right)$ é um processo de nascimento e morte com taxa de morte maior do que a taxa de nascimento, e assim, como pode ser visto com mais detalhes em [Dur12], sua medida invariante tem distribuição Geométrica, ou seja, é finita quase certamente. Como a condição inicial de $N_{t}^{+}$é vazia, temos como limitante de $N_{t}^{+}\left(0, \mu_{c}-\delta\right)$ a sua distribuição invariante, e assim concluímos que

$$
\frac{N_{t}^{+}\left(0, \mu_{c}-\delta\right)}{t} \rightarrow 0, \text { em probabilidade quando } t \rightarrow \infty .
$$

Desse modo, para $m \in \mathbb{N}^{*}$ fixo,

$$
\begin{aligned}
\mathbb{P}\left(\hat{\eta}_{t} \neq \eta_{t}^{+}\right) & \leq \mathbb{P}\left(\hat{\eta}_{t} \neq \eta_{t}^{+}, \hat{\eta}_{t}<m\right)+\mathbb{P}\left(\hat{\eta}_{t} \geq m\right) \\
& \leq \mathbb{P}\left(\hat{\eta}_{t} \neq \eta_{t}^{+}, \hat{\eta}_{t}<m, F_{t u}^{+}\left(\mu_{c}-\delta, 1\right)>u t I\left(\mu_{c}-\delta, 1\right)(1-\epsilon)\right) \\
& +\mathbb{P}\left(\hat{\eta}_{t} \geq m\right)+\mathbb{P}\left(F_{t u}^{+}\left(\mu_{c}-\delta, 1\right) \leq u t I\left(\mu_{c}-\delta, 1\right)(1-\epsilon)\right)
\end{aligned}
$$

Observamos que $\mathbb{P}\left(F_{t u}^{+}\left(\mu_{c}-\delta, 1\right) \geq u t I\left(\mu_{c}-\delta, 1\right)(1+\epsilon)\right)$ converge a zero quando $t$ diverge pelo Lema 2.2. Denotamos o evento

$$
Q:=\left\{F_{t u}^{+}\left(\mu_{c}-\delta, 1\right)>u t I\left(\mu_{c}-\delta, 1\right)(1-\epsilon)\right\} .
$$


Agora considere o conjunto $\left\{T_{1}^{*}, \ldots, T_{\hat{\eta}_{t}-1}^{*}\right\}$ como sendo os tempos de atribuição de indivíduos na espécie adicional no modelo $\mathcal{X}^{+}$. Definindo $\left\{H_{1}, \ldots, H_{\hat{\eta}_{t}}-1\right\}$ de modo que $H_{i}$ é o evento de que a atribuição de indivíduos no tempo $T_{i}^{*}$ foi em espécies diferentes em $\mathcal{X}^{+}$e $\mathcal{X}^{2}$, temos que

$$
\begin{aligned}
& \mathbb{P}\left(\hat{\eta}_{t} \neq \eta_{t}^{+}, \hat{\eta}_{t}<m, F_{t u}^{+}\left(\mu_{c}-\delta, 1\right)>u t I\left(\mu_{c}-\delta, 1\right)(1-\epsilon)\right) \\
\leq & \mathbb{P}\left(\bigcup_{j=1}^{\hat{\eta}_{t}-1} H_{j}, \hat{\eta}_{t}<m, F_{t u}^{+}\left(\mu_{c}-\delta, 1\right)>u t I\left(\mu_{c}-\delta, 1\right)(1-\epsilon)\right) \\
\leq & \sum_{l=1}^{m-1} \int_{t_{1}, \ldots, t_{l-1}} \mathbb{P}\left(\bigcup_{j=1}^{l-1} H_{j}, \hat{\eta}_{t}=l, T_{1}^{*}=t_{1}, \ldots, T_{l-1}^{*}=t_{l-1}, Q\right) \\
\leq & \sum_{l=1}^{m-1} \sum_{j=1}^{l-1} \int_{t_{1}, \ldots, t_{l-1}} \mathbb{P}\left(H_{j} \mid \hat{\eta}_{t}=l, T_{1}^{*}=t_{1}, \ldots, T_{l-1}^{*}=t_{l-1}, Q\right) \mathbb{P}\left(\hat{\eta}_{t}=l, T_{1}^{*}=t_{1}, \ldots, T_{l-1}^{*}=t_{l-1}, Q\right) .
\end{aligned}
$$

Agora, consideramos $\left\{\mathcal{X}_{t}^{+}\left(0, \mu_{c}\right), t>0\right\}$, o processo de evolução das espécies de $\mathcal{X}_{t}^{+}$com aptidão menor que que $\mu_{c}$. Esse processo foi estudado por Pinheiro em [Pin15] e por Swart e Formentin em [FS16] onde concluiu-se que a medida invariante do processo, denotada $\mathcal{I}$, existe. Ainda mais, definindo

$$
N_{\mathcal{I}}^{+}\left(0, \mu_{c}-\delta\right)
$$

como o número de espécies da medida invariante com aptidão menor que $\mu_{c}-\delta, N_{\mathcal{I}}^{+}\left(0, \mu_{c}-\delta\right)$ tem distribuição Geométrica com parâmetro $p \in(0,1)$, e portanto, finita quase certamente.

Assim, considerando o processo $\left\{\mathcal{X}_{t}^{\mathcal{I}}, t>0\right\}$ como sendo $\mathcal{X}^{+}$com distribuição inicial invariante $\mathcal{I}$ de aptidões abaixo de $\mu_{c}$, e o acoplando à $\mathcal{X}^{+}$convenientemente, como indicado abaixo, temos que

$$
\mathcal{X}_{t}^{+} \subseteq \mathcal{X}_{t}^{\mathcal{I}} .
$$

Tal propriedade vem do fato de que os nascimentos e as mortes dos dois processos são acoplados de modo que todos os nascimentos que ocorrerem em $\mathcal{X}_{t}^{+}$também ocorrerão em $\mathcal{X}_{t}^{\mathcal{I}}$, e também, como $\mathcal{X}_{t}^{\mathcal{I}}$ tem uma condição inicial com mais espécies, toda espécie extinta em $\mathcal{X}_{t}^{\mathcal{I}}$ será extinta em $\mathcal{X}_{t}^{+}$ou nem existirá em $\mathcal{X}_{t}^{+}$no tempo da extinção em $\mathcal{X}_{t}^{\mathcal{I}}$. Notamos agora que, definindo para $0 \leq a<b \leq 1$,

$$
\begin{aligned}
& F^{\mathcal{I}}:=\sum_{x \in \mathcal{X}^{\mathcal{I}}} f(x), \\
& F^{\mathcal{I}}(a, b):=\sum_{x \in \mathcal{X}^{\mathcal{I}}(a, b)} f(x),
\end{aligned}
$$

onde $\mathcal{I}(a, b):=\mathcal{I} \cap[a, b]$, para todo $\delta<\omega$ pequenos, temos que

$$
F^{\mathcal{I}}\left(0, \mu_{c}-\delta\right)=F^{\mathcal{I}}\left(0, \mu_{c}-\omega\right)+F^{\mathcal{I}}\left(\mu_{c}-\omega, \mu_{c}-\delta\right) .
$$

Como o número de espécies em $\mathcal{I}\left(0, \mu_{c}-\omega\right)$ é finito quase certamente, temos que

$$
F^{\mathcal{I}}\left(0, \mu_{c}-\omega\right)<\infty \text {, quase certamente. }
$$

Adicionalmente, pela suposição (1.5), sabemos que

$$
F^{\mathcal{I}}\left(\mu_{c}-\omega, \mu_{c}-\delta\right)<\infty, \text { quase certamente. }
$$

Notamos assim que $F^{\mathcal{I}}\left(0, \mu_{c}-\delta\right)$ é finita quase certamente além de não depender de $t$. 
Assim, para $j=1, \ldots, l-1$ temos que $t_{j} \geq u t$, e também,

$$
\begin{aligned}
& \mathbb{P}\left(H_{j} \mid \hat{\eta}_{t}=l, T_{1}^{*}=t_{1}, \ldots, T_{l-1}^{*}=t_{l-1}\right)= \\
& \mathbb{E}\left(\frac{F_{T_{j}^{*}}^{+}\left(0, \mu_{c}-\delta\right)}{F_{T_{j}^{*}}^{+}\left(0, \mu_{c}-\delta\right)+F_{T_{j}^{*}}^{2}} \mid \hat{\eta}_{t}=l, T_{1}^{*}=t_{1}, \ldots, T_{l-1}^{*}=t_{l-1}\right)= \\
& \mathbb{E}\left(\frac{F_{t_{j}}^{+}\left(0, \mu_{c}-\delta\right)}{F_{t_{j}}^{+}\left(0, \mu_{c}-\delta\right)+F_{t_{j}}^{2}}\right) \leq \\
& \mathbb{E}\left(\frac{F^{\mathcal{I}}\left(0, \mu_{c}-\delta\right)}{F^{\mathcal{I}}\left(0, \mu_{c}-\delta\right)+F_{u t}^{2}}\right) \rightarrow 0, \text { quando } t \rightarrow \infty .
\end{aligned}
$$

Notamos que a desigualdade de (3.18) vem do fato de $F_{t_{j}}^{+}\left(0, \mu_{c}-\delta\right)$ ser dominado estocasticamente por $F^{\mathcal{I}}\left(0, \mu_{c}-\delta\right)$, e como mostramos acima por (3.16) e (3.17), $F^{\mathcal{I}}\left(0, \mu_{c}-\delta\right)$ é finito quase certamente e não depende de $t$. Ainda mais, $F_{t_{j}}^{+}\left(0, \mu_{c}-\delta\right)$ é independente de $F_{t_{j}}^{2}$, da mesma forma que $F^{\mathcal{I}}\left(0, \mu_{c}-\delta\right)$ é independente de $F_{u t}^{2}$, que por sua vez,

$$
F_{u t}^{2} \rightarrow \infty \text {, quase certamente quando } t \rightarrow \infty \text {. }
$$

Por fim, $F_{u t}^{2} \leq F_{t_{j}}^{2}$ uma vez que para $j=1, \ldots, l-1$ temos que $t_{j} \geq u t$. As substituições feitas na desigualdade podem ser feitas pois os termos tem a forma

$$
\frac{x}{x+y}, x, y>0
$$

que é uma função crescente em $x$ e decrescente em $y$.

Denotando

$$
\rho(\delta, t, u):=\mathbb{E}\left(\frac{F^{\mathcal{I}}\left(0, \mu_{c}-\delta\right)}{F^{\mathcal{I}}\left(0, \mu_{c}-\delta\right)+F_{u t}^{2}}\right)
$$

e aplicando (3.18), temos que a expressão em (3.14) é limitada superiormente por

$$
\begin{aligned}
& \rho(\delta, t, u) \sum_{l=1}^{m-1} \sum_{j=1}^{l-1} \int_{t_{1}, \ldots, t_{l-1}} \mathbb{P}\left(\hat{\eta}_{t}=l, T_{1}^{*}=t_{1}, \ldots, T_{l-1}^{*}=t_{l-1}, Q\right) \\
\leq & \rho(\delta, t, u) \sum_{l=1}^{m-1} \int_{t_{1}, \ldots, t_{l-1}} \mathbb{P}\left(\hat{\eta}_{t}=l, T_{1}^{*}=t_{1}, \ldots, T_{l-1}^{*}=t_{l-1}, Q\right) \leq \rho(\delta, t, u) m \rightarrow 0,
\end{aligned}
$$

quando $t \rightarrow \infty$. Notamos que a desigualdade em (3.19) é verdadeira pois

$$
\sum_{l=1}^{m-1} \int_{t_{1}, \ldots, t_{l-1}} \mathbb{P}\left(\hat{\eta}_{t}=l, T_{1}^{*}=t_{1}, \ldots, T_{l-1}^{*}=t_{l-1}, Q\right) \leq 1,
$$

uma vez que se trata da soma da integral de uma probabilidade. Como todas as convergências acima valem para todo $m \in \mathbb{N}^{*}$, podemos tomar $m \rightarrow \infty$, e assim temos que

$$
\mathbb{P}\left(\hat{\eta}_{t} \geq m\right) \rightarrow 0 \text {, quando } t \rightarrow \infty \text {. }
$$

Assim, mostramos que todos os termos de (3.11) e (3.12) convergem a zero quando $t$ diverge, ou seja, mostramos que

$$
\mathbb{P}\left(\eta_{t}^{2} \neq \eta_{t}^{+}\right) \rightarrow 0, \text { quando } t \rightarrow \infty
$$

o que finaliza a demonstração. 
Demonstração. (Teorema 1.2) Por (3.8), basta considerarmos o termo

$$
\mathbb{P}\left(\left(I_{t}, A_{t}\right)=\left(I_{t}^{-}, A_{t}^{-}\right)=\left(I_{t}^{+}, A_{t}^{+}\right), G \cap\{Y=1\}\right)=\mathbb{E}\left(\frac{N_{t}^{-}-1}{N_{t}^{+}}\right) \mathbb{P}(G) .
$$

Notamos que $N_{t}^{+}=N_{t}^{-}+N_{\delta t}^{+}\left(\mu_{c}+\delta, 1\right)+N_{t}^{+}\left(0, \mu_{c}+\delta\right)$. Pela lei dos grandes números em processos pontuais de Poisson, sabemos que

$$
\frac{N_{t}^{-}}{t} \rightarrow\left(1-\mu_{c}-\delta\right)(1-\delta) r, \text { e } \frac{N_{\delta t}^{+}\left(\mu_{c}+\delta, 1\right)}{t} \rightarrow\left(1-\mu_{c}-\delta\right) \delta r \text { em prob. quando } t \rightarrow \infty .
$$

Ainda mais, como consequência do Lema 3.1,

$$
\frac{N_{t}^{+}\left(0, \mu_{c}+\delta\right)}{t} \rightarrow 2 \delta r \text { em probabilidade quando } t \rightarrow \infty .
$$

Desse modo,

$$
\frac{N_{t}^{-}-1}{N_{t}^{+}} \rightarrow \frac{\left(1-\mu_{c}-\delta\right)(1-\delta) r}{\left(1-\mu_{c}-\delta\right)(1-\delta) r+\left(1-\mu_{c}-\delta\right) \delta r+2 \delta r}
$$

em probabilidade quando $t \rightarrow \infty$.

Agora, condicionado à $\left\{\left(I_{t}, A_{t}\right)=\left(I_{t}^{-}, A_{t}^{-}\right)=\left(I_{t}^{+}, A_{t}^{+}\right)\right\}$, temos que $\eta_{t}^{+} \leq \eta_{t} \leq \eta_{t}^{-}$, e assim, para todo $\delta>0$ pequeno,

$$
\begin{aligned}
& \lim _{t \rightarrow \infty} \mathbb{P}\left(\eta_{t} \leq \eta_{t}^{-}\right) \geq \frac{\left(1-\mu_{c}-\delta\right)(1-\delta) r}{\left(1-\mu_{c}-\delta\right)(1-\delta) r+\left(1-\mu_{c}-\delta\right) \delta r+2 \delta r}, \\
& \lim _{t \rightarrow \infty} \mathbb{P}\left(\eta_{t}^{+} \leq \eta_{t}\right) \geq \frac{\left(1-\mu_{c}-\delta\right)(1-\delta) r}{\left(1-\mu_{c}-\delta\right)(1-\delta) r+\left(1-\mu_{c}-\delta\right) \delta r+2 \delta r} .
\end{aligned}
$$

Segue do Teorema 2.1 e Lema 2.3 que para $\lambda \geq 0$,

$$
\begin{aligned}
e^{-\lambda} \exp \left(\frac{(1-r)\left(e^{-\lambda}-1\right) f\left(a^{*}\right)}{I\left(\mu_{c}+\delta, 1\right)} \log \left(\frac{1}{u}\right)\right)= & \lim _{t \rightarrow \infty} \mathbb{E}\left(e^{-\lambda \eta_{t}^{+}\left(u, a^{*}\right)}\right) \leq \liminf _{t \rightarrow \infty} \mathbb{E}\left(e^{-\lambda \eta_{t}}\right) \leq \\
& \limsup _{t \rightarrow \infty} \mathbb{E}\left(e^{-\lambda \eta_{t}}\right) \leq \lim _{t \rightarrow \infty} \mathbb{E}\left(e^{-\lambda \eta_{t}^{-}\left(u, a^{*}\right)}\right)= \\
& e^{-\lambda} \exp \left(\frac{(1-r)\left(e^{-\lambda}-1\right) f\left(a^{*}\right)}{I\left(\mu_{c}-\delta, 1\right)} \log \left(\frac{1}{u}\right)\right) .
\end{aligned}
$$

Como a distribuição de $\eta_{t}$ não depende de $\delta$ e as expressões à esquerda e a direita de (3.22) tem o mesmo limite quando $\delta \rightarrow 0^{+}$, concluímos que

$$
\left(\eta_{t}, \frac{I_{t}}{t}, A_{t}\right) \rightarrow(N, U, V), \text { em distribuição quando } t \rightarrow \infty
$$

onde $U \sim \operatorname{Uniforme}(0,1), V \sim \operatorname{Uniforme}\left(\mu_{c}, 1\right)$ e $N \mid\{U=u, V=v\} \sim \operatorname{Poisson}\left(\frac{(1-r) f(v)}{\int_{\mu_{c}}^{1} f(u) d u} \log \left(\frac{1}{u}\right)\right)$. 


\section{Capítulo 4}

\section{Conclusões}

Nessa dissertação, estudamos o comportamento assintótico da transformada de Laplace do número de indivíduos de uma versão simplificada do modelo BAS (BASS), e a partir dela, analisamos o comportamento assintótico do modelo BAS através de acoplamentos entre o BAS e variações do BASS. Os resultados são obtidos para uma versão mais geral do BAS apresentado em [BAS16], pois a atribuição de indivíduos é feita através de qualquer função $f$ que satisfaça algumas propriedades. Além disso, o resultado apresentado fixa a idade da espécie sorteada. 


\section{Referências Bibliográficas}

[Ant99] H. Anton. Calculus: A New Horizon, 6th ed. New York: Wiley, pp. 324-3274, 1999. 12

[BAS16] I. Ben-Ari e R. B. Schinazi. A Stochastic Model for the Evolution of a Quasispecies. Journal of Statistical Physics, 162(2), 415-425, 2016. 1, 2, 27

[Dur12] R. Durret. Essentials of Stochastic Process. Springer, 2nd ed., 2012. 22

[FS16] M. Formentin e Jan M. Swart. The limiting shape of a full mailbox. ALEA Latin American Journal of Probability and Mathematical Statistics 13(2), 1151-1164, 2016. 23

[GMS11] H. Guiol, F. P. Machado e R. B. Schinazi. A stochastic model of evolution. Markov Process and Related Fields , 17:253-258, 2011. 2

[GMS13] H. Guiol, F. P. Machado e R. B. Schinazi. On a link between a species survival time in a evolution model and the bessel distributions. Brazilian Journal of Probability and Statistics, 27:201-209, 2013. 2

[Pin15] M. A. Pinheiro. Processos pontuais no modelo de Guiol-Machado-Schinazi de sobrevivência de espécies. Dissertação (Mestrado em Estatística) - Instituto de Matemática e Estatística, University of São Paulo, São Paulo, doi:10.11606/D.45.2016.tde-01062016-191528, 2015. 23 